\title{
FORMULATION OF NEW MODIFIED ALKYD RESINS AND THEIR APPLICATION IN THE FIELD OF SURFACE COATINGS
}

\author{
M. M. ELSHAHAWI ${ }^{*}$, A. ABOU SHEAISHAA*, M. ELSAYED *, AND F. ABD EL-HAI ** \\ Departments of Chemistry, Faculty of Science, *Ain Shams University, **Al-Azhar University, Cairo, Egypt
}

\begin{abstract}
Alkyd resins are considered modified polyester resins, which may be modified with many chemicals such as polybasic acids, polyhydric alcohols and/or fatty acids to produce resins with different properties and give the final surface coating formulations wide performance range. In this research, the soya bean based N,N-bis(2-hydroxyethyl)thiophene-2-carboxamide (HETCA)modified alkyd resins and $N, N$-bis(2-hydroxyethyl)furan-2-carboxamide (HEFCA)-modified alkyd resins are prepared by partial replacement of glycerol $(G)$ with each (HETCA) (I) and (HEFCA) (III), respectively, which are reacted as source of polyhydric alcohols, with both phthalic anhydride (PA) and soya bean oil fatty acids (SOFA), as source of polyacids .

The new HETCA-modified alkyd resins and previously prepared HEFCA-modified alkyd resins enter in final coating formulations and their performance are evaluated and compared using the international standard test methods (ASTM) and involved the measurement of phyisco-mechanical properties such as viscosity, drying time, specular gloss, pencil hardness, adhesion, flexibility. In general, the results show that the modification enhances both phyisco-mechanical and chemical properties. The corrosion resistance of alkyd resins improved by such HETCA and HEFCA-modification

Additionally, expected corrosion inhibitors such as morpholino(thiophen-2-yl)methanone (II) and morpholino(furan-2-yl) methanone (IV) are prepared and their anticorrosion effect is investigated by two ways. First, as an additive with unmodified (commercial) alkyd resin in zinc phosphate primer formulations. Second, as an additive with previously prepared HETCA-and HEFCA-modified alkyd resins in the same primer formulations. Both of corrosion inhibitors (II) and (IV) improve the corrosion resistance at certain dosages.
\end{abstract}

Keywords- anticorrosion coating, corrosion inhibitors, modified alkyd resin, N,N-bis(2-hydroxyethyl)thiophene2-carboxamide (HETCA), N,N-bis(2-hydroxyethyl)furan-2-carboxamide (HEFCA), morpholino(thiophen-2-yl)methanone, morpholino(furan-2-yl)methanone

\section{INTRODUCTION}

In the ongoing shift from solvent- to waterbased coatings, alkyds have stubbornly resisted the change, and for valid reasons. Solvent-based alkyds are cost efficient and versatile, with a long history of proven performance in architectural, industrial and specialty applications. They offer excellent adhesion, hardness, gloss and corrosion resistance. These resins are also highly viscous: they originate as solids, making it very difficult to formulate shelf-stable coatings without the addition of solvent. Despite numerous options, including water-reducible alkyds, modified alkyd dispersions and alkyd emulsions, only $10 \%$ of alkyd-based coatings are currently waterborne ${ }^{(1)}$. Challenges to widespread adoption include delayed hardness development, lower gloss and reduced corrosion protection.

N,N-bis(2-hydroxyethyl)furan-2-carboxamide (HEFCA)-modified alkyd resins are previously prepared by partial replacement of glycerol with N,N-bis(2-hydroxyethyl)furan2-carboxamide (HEFCA) which was reacted with Linseed oil fatty acids (LOFA) and phthalic anhydride (PA) without affecting the resin constant. The biological effect of HEFCA-modified alkyd resins are investigated and showed promising results ${ }^{(2)}$.

The modification of short and medium alkyd resins using perfluorinated urethane toluene isocyanate (PFUTI), and incorporates the modified resins in a set of paint formulations containing different ratios of zinc phosphate as an inhibitive pigment, achieves promising results and illustrates corrosion protective properties in various paint formulations and also in the paint formulation free of the inhibitive pigment, $\mathrm{s}$ qhows enhancement of the corrosion protection efficiencies by the improvement in the hydrophobicity of alkyd resin modified with PFUTI. In view of these results, minimizes or neglects the most expensive inhibitive pigments from an economical stand point ${ }^{(3)}$.

The usage of maleic anhydride as a partial replacement of phthalic anhydride in long oil modified alkyd resins is investigated. The usag- 
es of maleic anhydride as a partial replacement of phthalic anhydride at these resins as well as at the vegetable oils improve the characteristics of the film of paint, such as its hardness and its resistance against atmospheric and corrosive agents. Maleic anhydride influences also in the condensation time of alkyd resins. Usually, the maleic anhydride is added before the phthalic anhydride, because it links with the double bonds of the radicals of vegetable oils, raising the stereo chemical structure and helps to achieve, in a short time, the proper viscosity of the resin. Experiments have shown that the optimal quantity of maleic anhydride for achieving the desirable results is $2 \%{ }^{(4)}$.

Alkyd resins are recently prepared by using recycled poly (ethylene terephthalate), PET, and different vegetable oils. Glycolysis of PET waste using pentaerytheritol (PEr) was used to produce suitable hydroxyl oligomers, GPEr, for alkyd resin. The glycolysis was carried out in the presence of manganese acetate as a catalyst and m-cresol as a solvent at $220{ }^{\circ} \mathrm{C}$. Alkyd resins were prepared using phthalic anhydride, PET waste, glycerin, sunflower oil or linseed oil and ethylene glycol to produce PET-based alkyd resin. GPEr was used instead of PET waste to produce the second type of alkyd resin based on GPEr. The reactions were carried out in the presence of butylhydroxytin oxide as a catalyst to prepare alkyd resins. The curing characteristics of the resins produced were investigated. Corrosion resistivity based on salt spray and cathodic disbondment were evaluated for the cured alkyd

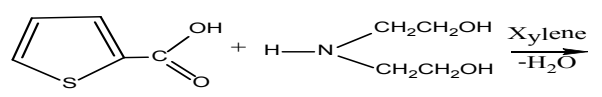

as organic coating for carbon steel ${ }^{(5)}$.

Recently microbial activity of N,N Di-hydroxyethyl-2-thiophenamide (HETCA) have been discussed and the studies showed promising results as biocidal coatings. Preparation of modified polyesteramides were also considered and found also improved the film performance and durability and lead to substantial antimicrobial growth control ${ }^{(6,7)}$.

\section{Experimental Materials}

The Soya bean fatty acid (SOFA) used is supplied by Guangrao Welfare Resin Factory, China, the phthalic anhydride (PA) and the diethanolamine (DEA) are obtained from Sdfine Indian. The Glycerin are obtained from El Gomhouria Co., Egypt. Thiophene -2-carboxylic acid is obtained from SIGMA Chemical Co., USA. Furan-2-carboxylic acid is obtained from SIGMA-ALDRICH Co., United Kingdom.

\section{METHODS AND TECHNIQUES}

1. Preparation of $\mathrm{N}, \mathrm{N}$-bis(2-hydroxyethyl) thiophene-2-carboxamide (HETCA) I ${ }^{(6,7)}$

A mixture of thiophene -2-carboxylic acid $(0.1$ mole $=12.815 \mathrm{gm})$, freshly distilled diethanolamine $(0.1 \mathrm{~mole}=10.5 \mathrm{gm})$, and $21 \mathrm{ml}$ xylene as a solvent were placed in $250 \mathrm{ml}$ round-bottomed flask fitted with Dean and Stark apparatus, The reaction was allowed to reflux until the theoretical amount of water $(0.1$ mole $=1.8 \mathrm{gm}$ or $1.8 \mathrm{ml}$ )was collected. The mixture was allowed to cool where a clear pale yellow viscous material was obtained.

Equation :

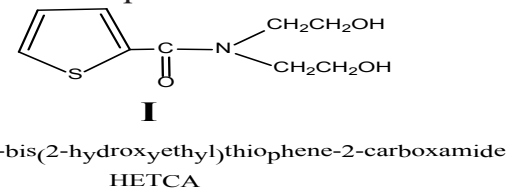

flask fitted with Dean and Stark apparatus, The reaction was allowed to reflux until the theoretical amount of water $(0.1$ mole $=1.8 \mathrm{gm}$ or $1.8 \mathrm{ml})$ was collected. The mixture was allowed to cool where a brown viscous material was obtained.

Equation :

\section{2- Preparation of N,N-bis(2-hydroxyethyl) furan-2-carboxamide (HEFCA) III (2)}

A mixture of furan-2-carboxylic acid (0.1 mole $=11.208 \mathrm{gm})$, freshly distilled diethanolamine $(0.1$ mole $=10.5 \mathrm{gm})$, and $21 \mathrm{ml}$ xylene as a solvent were placed in $250 \mathrm{ml}$ round-bottomed

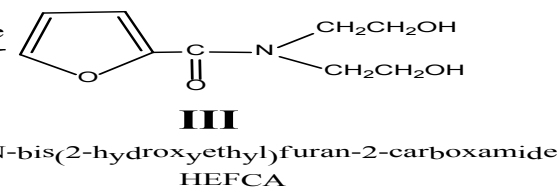


3- Preparation Of Alkyd Resins via solvent process $^{(8)}$ :

The preparation of various modified alkyd resins carried out as shown in the following equations:

By partial replacement of Glycerol (G) with each of following compounds:

N,N-bis(2-hydroxyethyl)thiophene-2-carboxamide (HETCA) I

N,N-bis(2-hydroxyethyl)furan-2-carboxamide (HEFCA) III

As the ingredient source of the polyol, while Phthalic anhydride (PA) and Soya bean oil fatty acid (SOFA) is the source of the polybasic acid, in the presence of $10 \%$ xylene of their volume, the whole ingredient was placed in $250 \mathrm{ml}$ roundbottomed flask fitted with Dean Stark apparatus.

It should be noted that within each set of formulation, the total number of acid and hydroxyl equivalents for various runs were kept constant and the partial replacement of Glycerol with each compound " I" and "III" is listed in table (1).

Table (1): List of hydroxyl equivalent of different runs

\begin{tabular}{|l|l|l|}
\hline Resin & $\mathrm{G}$ & HETCA or HEFCA \\
\hline $\mathrm{B}_{\mathrm{x}}$ & 1.00 & 0.00 \\
$\mathrm{~T}_{\mathrm{x} 1}$ or $\mathrm{F}_{\mathrm{x} 1}$ & 0.95 & 0.05 \\
$\mathrm{~T}_{\mathrm{x} 2}$ or $\mathrm{F}_{\mathrm{x} 2}$ & 0.90 & 0.10 \\
$\mathrm{~T}_{\mathrm{x} 3}$ or $\mathrm{F}_{\mathrm{x} 3}$ & 0.80 & 0.20 \\
$\mathrm{~T}_{\mathrm{x} 4}$ or $\mathrm{F}_{\mathrm{x} 4}$ & 0.70 & 0.30 \\
\hline
\end{tabular}

$X=0,1,2$ or 3 at $0 \%, 10 \%, 20 \%$ or $30 \%$ excess$\mathrm{OH}$ respectively

B: Unmodified Alkyd Resin (Blank)

T: HETCA-modified Alkyd Resin

F: HEFCA-modified Alkyd Resin
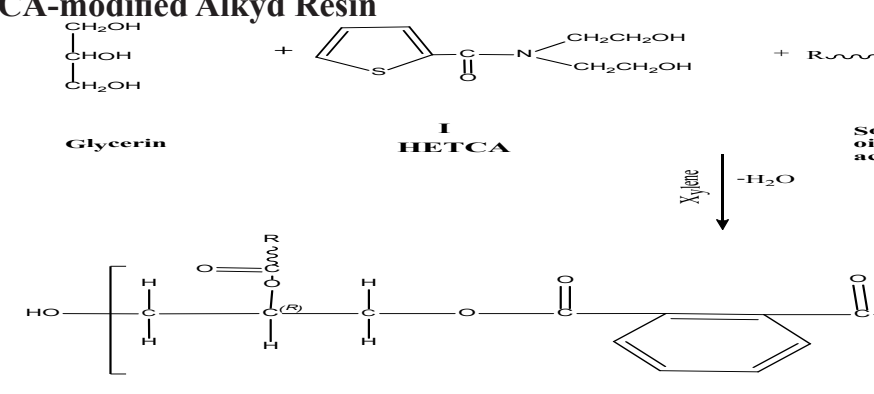

il.

3.2.Preparation Of HETCA-Modified Alkyd Resins Based On Soyabean Oil Fatty Acid
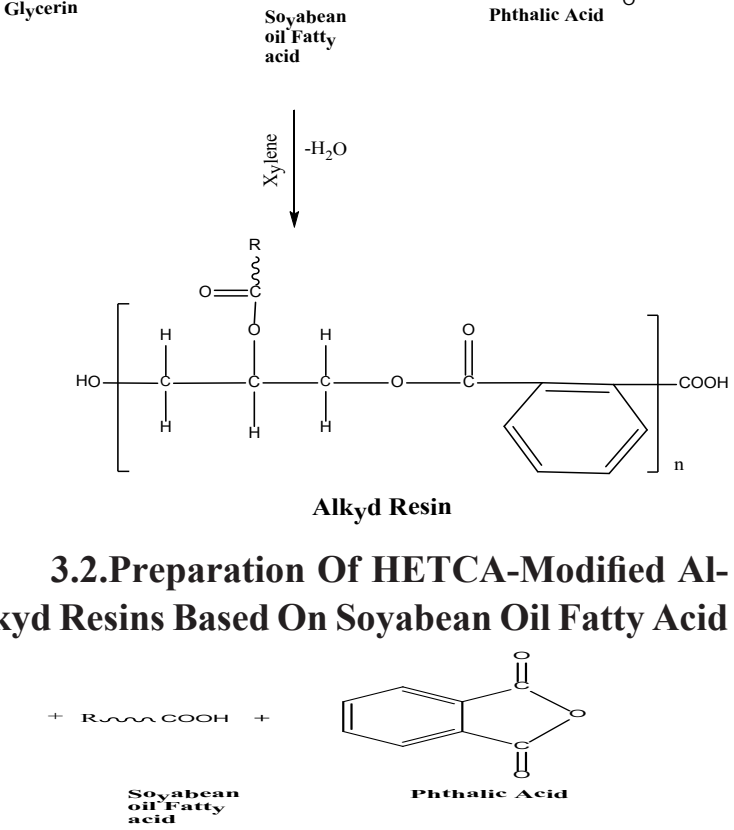

Alkyd Resin

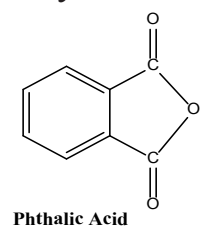

2- Gradual increase in the percentage of the
modifier. The modifier is partially replaces the
hydroxyl equivalent of Glycerol used in formu-
lation. Such replacement is based on the hydrox-
yl equivalent constant and the second subscript
numbers $1,2,3$ and 4 are given after the formu-
lation numbers to indicate $5 \%, 10 \%, 20 \%$ and
$30 \%$ modification partial replacement, respec-
tively. for various modified alkyd resin formulations.

These formulations cover a wide range of various excess hydroxyl contents $(0 \%, 10 \%$, $20 \%$, and $30 \%$ excess-OH).It is important to compound and is introduced for comparison purpose. This formulation will be B0, B1, B2 or B3 to indicate excess-OH of $0 \%, 10 \%, 20 \%$ or $30 \%$ respectively. tively.

3.1. Preparation of Unmodified Alkyd Resins ${ }^{(8)}$ 
M. M. ELSHAHAWI, et al.

Table (2): Resin constants for HETCA -modified alkyd resins

\begin{tabular}{|c|c|c|c|c|c|c|c|c|c|}
\hline Resin No. & $\begin{array}{l}\text { Excess - } \\
\mathrm{OH}(\%)\end{array}$ & Ingredients & $\mathrm{E}$ & $\mathrm{F}$ & $e_{0}$ & $e_{A}$ & $\mathrm{e}_{\mathrm{B}}$ & $\mathrm{R}$ & $\mathrm{H}_{2} \mathrm{O}$ off $(\mathrm{ml})$ \\
\hline \multirow{4}{*}{$\mathrm{T}_{01-04}$} & \multirow{4}{*}{0} & G & 30.7 & 3 & \multirow{2}{*}{0.520} & & \multirow{2}{*}{0.520} & \multirow{4}{*}{1.00} & \multirow{4}{*}{6.7} \\
\hline & & HETCA & 107.6 & 2 & & & & & \\
\hline & & FA & 280 & 1 & 0.222 & \multirow{2}{*}{0.519} & & & \\
\hline & & PA & 74.1 & 2 & 0.297 & & & & \\
\hline \multirow{4}{*}{$\mathrm{T}_{11-14}$} & \multirow{4}{*}{10} & G & 30.7 & 3 & \multirow{2}{*}{0.600} & & \multirow{2}{*}{0.600} & \multirow{4}{*}{1.10} & \multirow{4}{*}{6.7} \\
\hline & & HETCA & 107.6 & 2 & & & & & \\
\hline & & FA & 280 & 1 & 0.200 & \multirow{2}{*}{0.546} & & & \\
\hline & & PA & 74.1 & 2 & 0.346 & & & & \\
\hline \multirow{4}{*}{$\mathrm{T}_{21-24}$} & \multirow{4}{*}{20} & G & 30.7 & 3 & \multirow{2}{*}{0.716} & & \multirow{2}{*}{0.716} & \multirow{4}{*}{1.20} & \multirow{4}{*}{6.8} \\
\hline & & HETCA & 107.6 & 2 & & & & & \\
\hline & & FA & 280 & 1 & 0.164 & \multirow{2}{*}{0.596} & & & \\
\hline & & PA & 74.1 & 2 & 0.432 & & & & \\
\hline \multirow{4}{*}{$\mathrm{T}_{31-34}$} & \multirow{4}{*}{30} & G & 30.7 & 3 & \multirow{2}{*}{0.879} & & \multirow{2}{*}{0.879} & \multirow{4}{*}{1.30} & \multirow{4}{*}{7.1} \\
\hline & & HETCA & 107.6 & 2 & & & & & \\
\hline & & FA & 280 & 1 & 0.111 & \multirow{2}{*}{0.679} & & & \\
\hline & & PA & 74.1 & 2 & 0.568 & & & & \\
\hline
\end{tabular}

G:glycerol; HETCA:N,N-bis(2-hydroxyethyl)thiophene-2-carboxamide; PA: Phthalic Anhydride; FA: Soya bean fatty acid E: Equivalent Weight; $e_{A}$ : Number of acid equivalent $; e_{B}$ : Number of hydroxyl equivalent; $\mathrm{e}_{0}$ : Total equivalent present at the start of the reaction; $\mathrm{F}$ : Functionality ; R:Ratio of total-OH groups to total-COOH groups $\left(=\mathrm{e}_{\mathrm{B}} / \mathrm{e}_{\mathrm{A}}\right) ; \mathrm{H} 2 \mathrm{O}$ off $(\mathrm{ml})$ : Number of water millimeters at which the reaction will be Completed $\left(=\mathrm{e}_{\text {acid }} \times 18+\mathrm{e}_{\text {anhydride }} \times 9\right)$

3.2. Preparation Of HEFCA-Modified Alkyd Resins Based On Soyabean Oil Fatty Acid $^{(2)}$

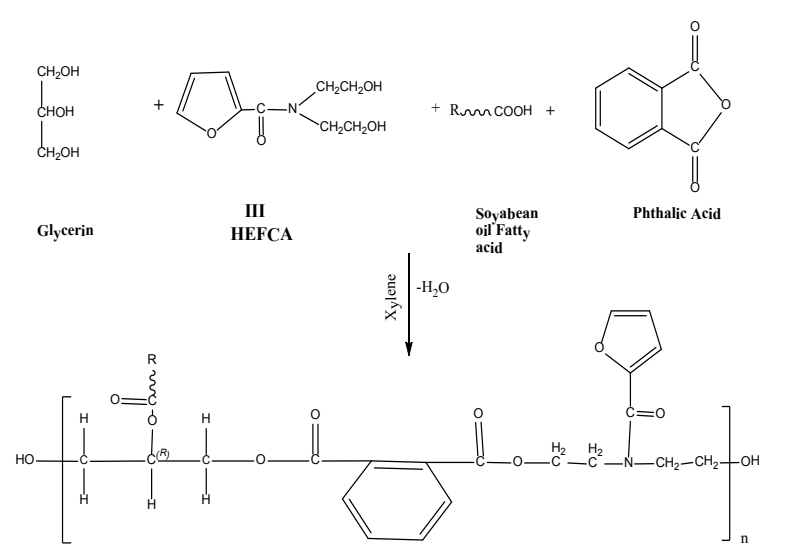

HEFCA-modified alkyd resin

\section{4-Preparation of morpholino(thiophen-2- yl)methanone II}

A mixture of thiophene-2-carboxylic acid $(0.1 \mathrm{~mole}=12.815 \mathrm{gm})$, freshly distilled diethanolamine $(0.1 \mathrm{~mole}=10.5 \mathrm{gm})$, and $21 \mathrm{ml}$ xylene as a solvent were placed in $250 \mathrm{ml}$ round-bottomed flask fitted with Dean and Stark apparatus, The reaction was allowed to reflux until the theoretical amount of water $(0.2 \mathrm{~mole}=3.6 \mathrm{gm}$ or $3.6 \mathrm{ml}$ )was collected. The mixture was allowed to cool where a brown viscous material was obtained.

Equation :

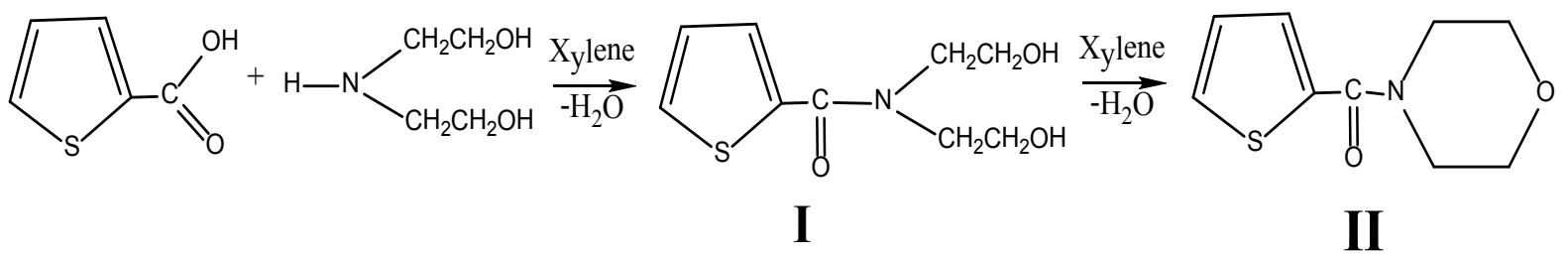

Morpholino(thiophen-2-yl)methanone 
FORMULATION OF NEW MODIFIED ALKYD RESINS AND THEIR APPLICATION

Table (3): Resin constants for HEFCA -modified alkyd resins

\begin{tabular}{|c|c|c|c|c|c|c|c|c|c|}
\hline Resin No. & $\begin{array}{c}\text { Excess } \\
-\mathrm{OH} \\
(\%)\end{array}$ & Ingredients & $\mathrm{E}$ & $\mathrm{F}$ & $\mathrm{e}_{0}$ & $\mathrm{e}_{\mathrm{A}}$ & $\mathrm{e}_{\mathrm{B}}$ & $\mathrm{R}$ & $\begin{array}{c}\mathrm{H}_{2} \mathrm{O} \text { off } \\
(\mathrm{ml})\end{array}$ \\
\hline \multirow{4}{*}{$\mathrm{T}_{01-04}$} & \multirow{4}{*}{0} & G & 30.7 & 3 & \multirow{2}{*}{0.520} & & \multirow{2}{*}{0.520} & \multirow{4}{*}{1.00} & \multirow{4}{*}{6.7} \\
\hline & & HEFCA & 99.5 & 2 & & & & & \\
\hline & & FA & 280 & 1 & 0.222 & \multirow{2}{*}{0.519} & & & \\
\hline & & PA & 74.1 & 2 & 0.297 & & & & \\
\hline \multirow{4}{*}{$\mathrm{T}_{11-14}$} & \multirow{4}{*}{10} & $\mathrm{G}$ & 30.7 & 3 & \multirow{2}{*}{0.600} & & \multirow{2}{*}{0.600} & \multirow{4}{*}{1.10} & \multirow{4}{*}{6.7} \\
\hline & & HEFCA & 99.5 & 2 & & & & & \\
\hline & & FA & 280 & 1 & 0.200 & \multirow{2}{*}{0.546} & & & \\
\hline & & PA & 74.1 & 2 & 0.346 & & & & \\
\hline \multirow{4}{*}{$\mathrm{T}_{21-24}$} & \multirow{4}{*}{20} & $\mathrm{G}$ & 30.7 & 3 & \multirow{2}{*}{0.716} & & \multirow{2}{*}{0.716} & \multirow{4}{*}{1.20} & \multirow{4}{*}{6.8} \\
\hline & & HEFCA & 99.5 & 2 & & & & & \\
\hline & & FA & 280 & 1 & 0.164 & \multirow{2}{*}{0.596} & & & \\
\hline & & $\mathrm{PA}$ & 74.1 & 2 & 0.432 & & & & \\
\hline \multirow{4}{*}{$\mathrm{T}_{31-34}$} & \multirow{4}{*}{30} & $\mathrm{G}$ & 30.7 & 3 & & & & \multirow{4}{*}{1.30} & \multirow{4}{*}{7.1} \\
\hline & & HEFCA & 99.5 & 2 & 0.879 & & 0.879 & & \\
\hline & & FA & 280 & 1 & 0.111 & \multirow{2}{*}{0.679} & & & \\
\hline & & PA & 74.1 & 2 & 0.568 & & & & \\
\hline
\end{tabular}

G:glycerol; HEFCA:N,N-bis(2-hydroxyethyl)furan-2-carboxamide; PA: Phthalic Anhydride; FA: Soya bean fatty acid $E$ : Equivalent Weight; $e_{A}$ : Number of acid equivalent $; e_{B}$ : Number of hydroxyl equivalent; $e_{0}$ : Total equivalent present at the start of the reaction; F: Functionality ; R:Ratio of total-OH groups to total-COOH groups $\left(=\mathrm{e}_{\mathrm{B}} / \mathrm{e}_{\mathrm{A}}\right) ; \mathrm{H} 2 \mathrm{O}$ off $(\mathrm{ml})$ : Number of water millimeters at which the reaction will be Completed $\left(=\mathrm{e}_{\text {acid }} \times 18+\mathrm{e}_{\text {anhydride }} \times 9\right)$

5-Preparation of furan-2-yl(morpholino) reaction was allowed to reflux until the theoretimethanone IV cal amount of water $(0.2$ mole $=3.6 \mathrm{gm}$ or $3.6 \mathrm{ml})$

A mixture of furan-2-carboxylic acid (0.1 mole $=11.208 \mathrm{gm})$, freshly distilled diethanolamine $(0.1 \mathrm{~mole}=10.5 \mathrm{gm})$, and $21 \mathrm{ml}$ xylene as a solvent were placed in $250 \mathrm{ml}$ round-bottomed flask fitted with Dean and Stark apparatus, The<smiles>[Y14][C@@H](O)c1ccc(C(=O)N2CCOCC2)o1</smiles>

Structural features of each of the previous III IV compounds is confirmed by IR spectroscopy which tabulated in table (4, 5, 6 and 7) and Figures (1, 2, 3 and 4) respectively

Table (4): FTIR spectra of N,N-bis (2-hydroxyethyl) thiophene-2-carboxamide (HETCAI

\begin{tabular}{|l|l|}
\hline Functional group & IR peak $\left(\mathrm{cm}^{-1}\right)$ \\
\hline $\mathrm{OH}$ & $3363($ Broad band $)$ \\
\hline $\mathrm{CH}$ aromatic & 3099 \\
\hline $\mathrm{CH}$ aliphatic & 2955 \\
\hline $\mathrm{CO}$ amide & 1705 \\
\hline
\end{tabular}
was collected. The mixture was allowed to cool where a brown viscous material was obtained.

Equation :

Furan-2-yl(morpholino)methanone

Spectral analysis of prepared compounds:

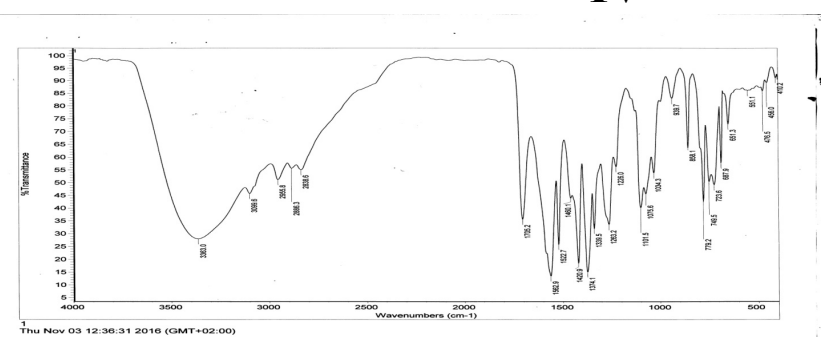

Figure (1) FTIR spectra of N,N-bis(2-hydroxyethyl) thiophene-2-carboxamide (HETCA) I 
Table (5): Spectral analysis of morpholino (thiophen-2-yl) methanone II

\begin{tabular}{|l|l|}
\hline Functional group & IR peak $\left(\mathrm{cm}^{-1}\right)$ \\
\hline $\mathrm{OH}$ & diminished \\
\hline $\mathrm{CH}$ aromatic & 3097 \\
\hline $\mathrm{CH}$ aliphatic & 2943 \\
\hline $\mathrm{CO}$ amide & 1708 \\
\hline
\end{tabular}

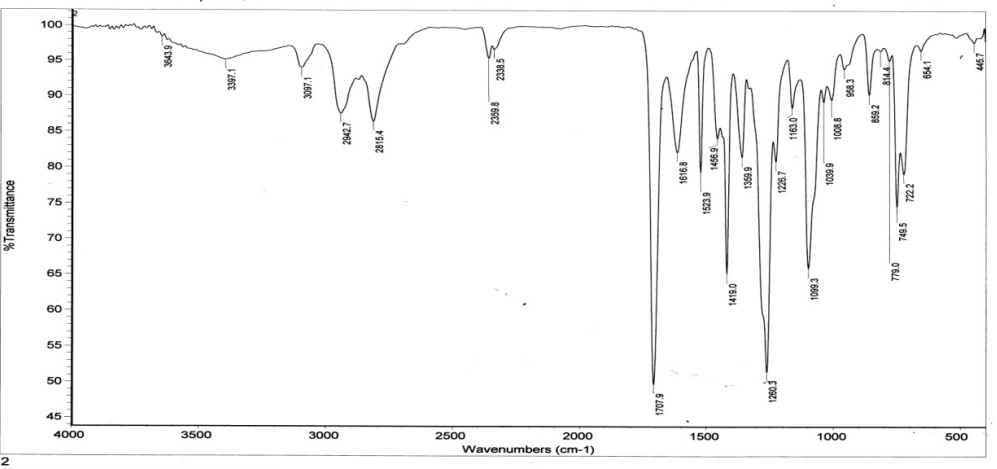

Table (6): Spectral analysis of $\mathrm{N}, \mathrm{N}-$

Figure (2) FTIR spectra of morpholino(thiophen-2-yl)methanone II bis(2-hydroxyethyl)furan2-carboxamide (HEFCA) III

\begin{tabular}{|l|l|}
\hline Functional group & IR peak $\left(\mathrm{cm}^{-1}\right)$ \\
\hline $\mathrm{OH}$ & $\begin{array}{l}3391(\text { Broad } \\
\text { band })\end{array}$ \\
\hline $\mathrm{CH}$ aromatic & 3050 \\
\hline $\mathrm{CH}$ aliphatic & 2957 \\
\hline $\mathrm{CO}$ amide & 1719 \\
\hline
\end{tabular}

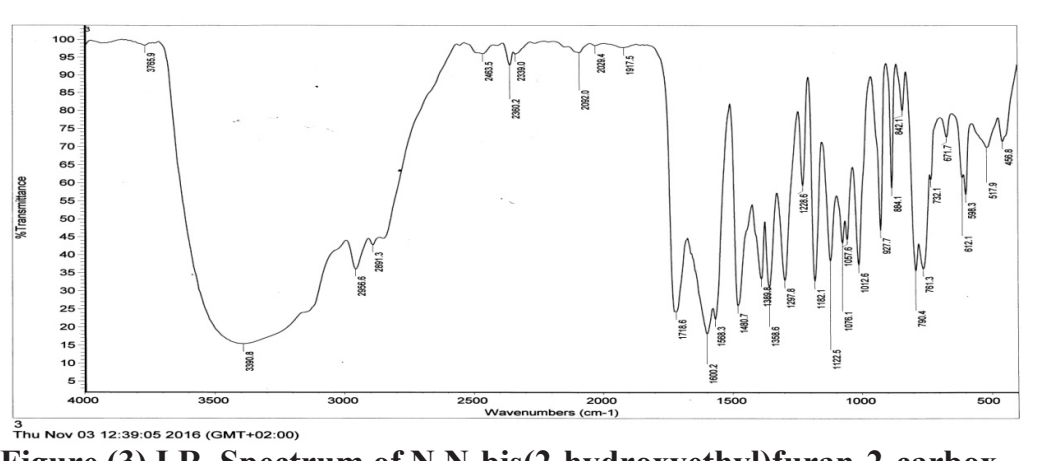

Figure (3) I.R. Spectrum of N,N-bis(2-hydroxyethyl)furan-2-carboxamide (HEFCA) III

Table (7): Spectral analysis of furan-2-yl(morpholino)methanone IV

\begin{tabular}{|l|l|}
\hline Functional group & IR peak $\left(\mathrm{cm}^{-1}\right)$ \\
\hline $\mathrm{OH}$ & diminished \\
\hline $\mathrm{CH}$ aromatic & 3024 \\
\hline $\mathrm{CH}$ aliphatic & 2955 \\
\hline $\mathrm{CO}$ amide & 1734 \\
\hline
\end{tabular}

\section{Method of testing and evaluation:}

The structure of the prepared N,N-bis(2-hydroxyethyl)thiophene-2-carboxamide (HETCA) I, morpholino(thiophen-2-yl)methanone II, N,N-bis(2-hydroxyethyl)furan-2-carboxamide (HEFCA) III and furan-2-yl(morpholino)methanone IV was confirmed by FTIR and showed in Figure 1, 23 and 4 respectively.

All resins are adjusted to solid content $=60$ \% (according to ASTM Method, D1644 - 01 (Reapproved 2012) and the following tests were carried out according to international standards:

Test method for color of transparent liquid, ASTM method, D1544 - 04 (Reapproved 2010).

Test method of viscosity, ASTM method, D4287 - 00 (Reapproved 2014).

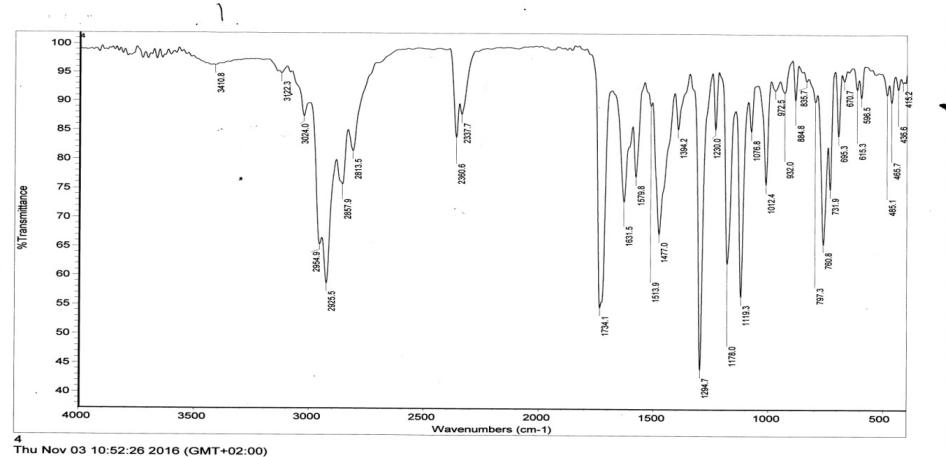

Figure (4) I.R. Spectrum of furan-2-yl(morpholino)methanone IV Preparation of glass panels, ASTM method, D3891 - 96

Water resistance of dried films, ASTM method, D870 - 15

Alkali resistance of dried films, Indian Standard Specification, 158(1950)

Acid resistance of dried films, Indian Standard Specification, 159(1950)

Solvent resistance of dried films, ASTM method, D2792-69 (Reapproved 2015)

Flexibility by conical mandrel bending tester, ASTM method, D522/D522M - 13

Pencil Hardness measurements, ASTM method, D3363 - 05 (Reapproved 2011) e2

Adhesion by cross-cut adhesion, ASTM method, 3359 - 09e2 
Specular gloss, ASTM method, D523 - 14

Impact test, ASTM method, D2794 - 93 (Reapproved 2010)

Measurement of film thickness, ASTM method, D1005 - 95 (Reapproved 2013)

Preparation of steel panels for testing purpose, ASTM method, D609 - 00 (Reapproved 2012)

Corrosion Resistance test, ASTM method, D2803 - 09 (Reapproved 2015), Procedure B (ISO 4623)

Determination of degree of blistering, ASTM method, D714 - 02 (Reapproved 2009)

\section{RESULTS AND DISCUSSION}

During the course of the preparation, it was felt of interest to determine the reaction time, the reaction completion. This is indicated by watching the amount of water collected in the trap and compared its amount by that calculated. The data obtained are given in the following tables ( 8 and 9) and Figures (5 and 6 )

Table (8) The reaction time of formation of HETCA-modified alkyd resins

\begin{tabular}{|c|c|c|c|}
\hline $\begin{array}{l}\text { Resin } \\
\text { Code }\end{array}$ & $\begin{array}{c}\text { Excess- } \\
\mathrm{OH}^{2}\end{array}$ & $\begin{array}{c}\text { HETCA } \\
\text { replacement } \\
(\%)\end{array}$ & $\begin{array}{c}\text { Reaction } \\
\text { Time } \\
\text { (minutes) }\end{array}$ \\
\hline B0 & \multirow{5}{*}{$0 \%$} & 0 & 720 \\
\hline T01 & & 5 & 600 \\
\hline T02 & & 10 & 300 \\
\hline T03 & & 20 & 90 \\
\hline T04 & & 30 & 240 \\
\hline B1 & \multirow{5}{*}{$10 \%$} & 0 & 360 \\
\hline T11 & & 5 & 300 \\
\hline $\mathrm{T} 12$ & & 10 & 120 \\
\hline T13 & & 20 & 70 \\
\hline $\mathrm{T} 14$ & & 30 & 180 \\
\hline B2 & \multirow{5}{*}{$20 \%$} & 0 & 480 \\
\hline $\mathrm{T} 21$ & & 5 & 70 \\
\hline $\mathrm{T} 22$ & & 10 & 90 \\
\hline $\mathrm{T} 23$ & & 20 & 50 \\
\hline $\mathrm{T} 24$ & & 30 & 60 \\
\hline B3 & \multirow{5}{*}{$30 \%$} & 0 & 300 \\
\hline T31 & & 5 & 90 \\
\hline T32 & & 10 & 70 \\
\hline T33 & & 20 & 60 \\
\hline T34 & & 30 & 60 \\
\hline
\end{tabular}

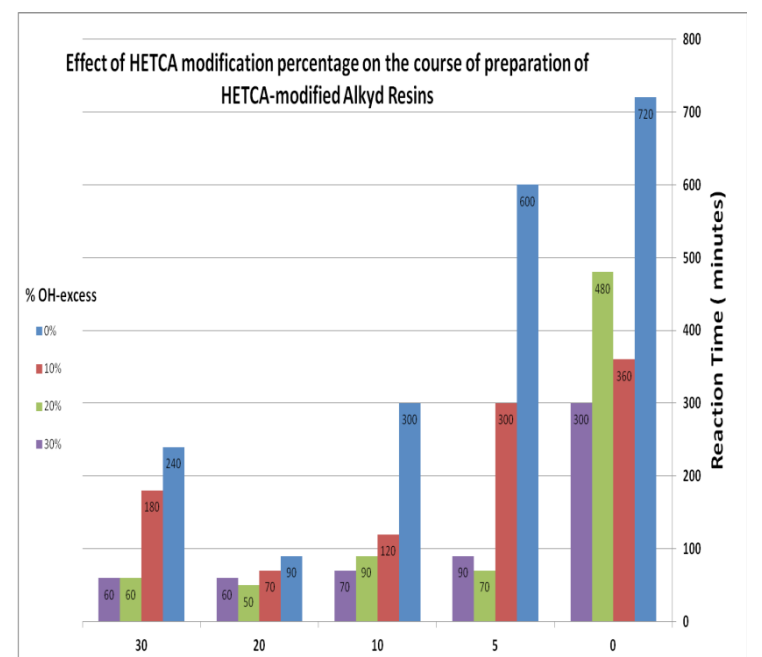

Fig (5): The reaction time of formation of HETCA-modified alkyd resins

Table (9) The reaction time of formation of HEF-

\begin{tabular}{|c|c|c|c|}
\hline \multicolumn{2}{|c|}{ CA-modified } & \multicolumn{2}{|c|}{ alkyd } \\
\hline $\begin{array}{l}\text { Resin } \\
\text { Code }\end{array}$ & $\begin{array}{l}\text { Excess- } \\
\mathrm{OH} \%\end{array}$ & $\begin{array}{l}\text { HEFCA } \\
\text { replacement } \\
(\%)\end{array}$ & $\begin{array}{l}\text { Reaction Time } \\
\text { (hours) }\end{array}$ \\
\hline B0 & & 0 & 720 \\
\hline F01 & & 5 & 240 \\
\hline F02 & $0 \%$ & 10 & 105 \\
\hline F03 & & 20 & 50 \\
\hline F04 & & 30 & 220 \\
\hline B1 & & 0 & 360 \\
\hline F11 & & 5 & 210 \\
\hline F12 & $10 \%$ & 10 & 220 \\
\hline F13 & & 20 & 210 \\
\hline F14 & & 30 & 210 \\
\hline $\mathrm{B} 2$ & & 0 & 480 \\
\hline F21 & & 5 & 55 \\
\hline F22 & $20 \%$ & 10 & 120 \\
\hline F23 & & 20 & 140 \\
\hline F24 & & 30 & 60 \\
\hline B3 & & 0 & 300 \\
\hline F31 & & 5 & 100 \\
\hline F32 & $30 \%$ & 10 & 60 \\
\hline F33 & & 20 & 55 \\
\hline F34 & & 30 & 45 \\
\hline
\end{tabular}

The reaction rate for various resins increases by the increasing of the percentage of modifier and also increases with the increase of excess$\mathrm{OH}$ percentage.

\section{Evaluation Studies}

Following the preparation of two series of 


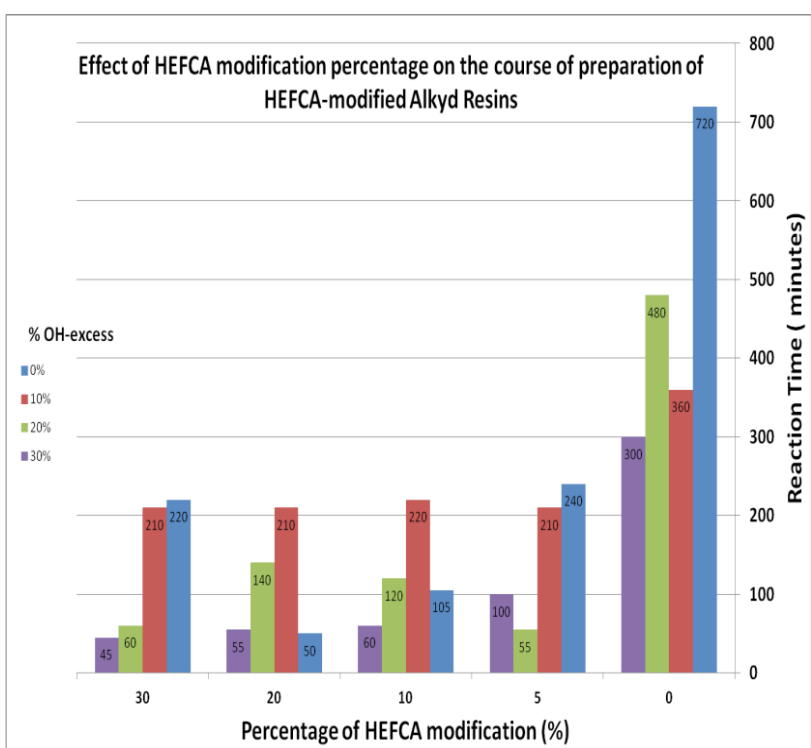

Fig (6): The reaction time of formation of HEFCAmodified alkyd resins

modified alkyd resins, the solid content of all formulations are adjusted to $60 \%$ solids. The drier combinations are added as mixed combinations by $3 \%$ from the weight of resin. The mixed driers consist of the following components:

Table (10)

\begin{tabular}{|l|l|}
\hline Component & $\%$ \\
\hline Cobalt Octoate $10 \%$ & 20 \\
\hline Calcium Octoate $10 \%$ & 45 \\
\hline Zirconium Octoate $12 \%$ & 29 \\
\hline Anti skinning agent & 6 \\
\hline Total & 100 \\
\hline
\end{tabular}

And, Varnish formula will be,

Table (11)

\begin{tabular}{|l|l|}
\hline Component & $\%$ \\
\hline $\begin{array}{l}\text { Alkyd resin or Modified alkyd resin (Solid } \\
\text { content=100\%) }\end{array}$ & 60 \\
\hline Xylene & 37 \\
\hline Mixed Drier & 3 \\
\hline Total & 100 \\
\hline
\end{tabular}

Table ( 12 ): Viscosity, Color, Drying time Characteristics of various HETCA-Modified Alkyd Resins

\begin{tabular}{|c|c|c|c|c|c|c|c|c|c|c|}
\hline \multirow[b]{3}{*}{$\begin{array}{l}\text { Resin } \\
\text { Code }\end{array}$} & \multirow[b]{3}{*}{$\begin{array}{l}\text { Excess- } \\
\text { ОН (\%) }\end{array}$} & \multirow{3}{*}{$\begin{array}{c}\text { HETCA } \\
\text { Replacement } \\
(\%)\end{array}$} & \multirow{3}{*}{$\begin{array}{c}\text { Viscosity } \\
(\mathrm{mPa} . \mathrm{s}) \\
\text { at solid } \\
\text { content }= \\
70 \%\end{array}$} & \multirow{3}{*}{$\begin{array}{c}\text { Air } \\
\text { Drying } \\
\text { (HD } \\
\text { Time) } \\
\text { (hr) }\end{array}$} & \multicolumn{5}{|c|}{ Stoving dry } & \multirow{3}{*}{$\begin{array}{c}\text { Color } \\
\text { (Gardner) }\end{array}$} \\
\hline & & & & & \multicolumn{2}{|c|}{$110 \mathrm{C}$} & \multicolumn{3}{|c|}{$120 \mathrm{C}$} & \\
\hline & & & & & $1 \mathrm{hr}$ & $2 \mathrm{hrs}$ & $1 \mathrm{hr}$ & $2 \mathrm{hrs}$ & $\begin{array}{c}\text { Extra } \\
\text { Stoving } \\
\text { Drying time } \\
\text { (hrs) }\end{array}$ & \\
\hline B0 & \multirow{5}{*}{$0 \%$} & $\mathbf{0}$ & 50 & 13 & VST & HD & - & - & - & 8 \\
\hline T01 & & 5 & 80 & 10 & VST & HD & - & - & - & $>18$ \\
\hline T02 & & 10 & 85 & $>24$ & ST & VST & HD & - & - & $>18$ \\
\hline T03 & & 20 & 100 & $>24$ & $\mathrm{~T}$ & $\mathrm{~T}$ & VST & VST & 3 & $>18$ \\
\hline T04 & & 30 & 100 & $>24$ & $\mathrm{~T}$ & $\mathrm{~T}$ & $\mathrm{~T}$ & $\mathrm{~T}$ & $>12$ & $>18$ \\
\hline B1 & \multirow{5}{*}{$10 \%$} & $\mathbf{0}$ & 140 & 11 & VST & HD & - & - & - & 8 \\
\hline T11 & & 5 & 100 & 8 & VST & HD & - & - & - & $>18$ \\
\hline T12 & & 10 & 150 & 9 & VST & HD & - & - & - & $>18$ \\
\hline T13 & & 20 & 250 & $>24$ & $\mathrm{~T}$ & $\mathrm{~T}$ & $\mathrm{~T}$ & $\mathrm{~T}$ & 12 & $>18$ \\
\hline T14 & & 30 & 750 & $>24$ & $\mathrm{~T}$ & $\mathrm{~T}$ & $\mathrm{~T}$ & $\mathrm{~T}$ & $>12$ & $>18$ \\
\hline B2 & \multirow{5}{*}{$20 \%$} & $\mathbf{0}$ & 450 & 7 & VST & HD & - & - & - & 9 \\
\hline T21 & & 5 & 675 & 6 & HD & - & - & - & - & $>18$ \\
\hline $\mathrm{T} 22$ & & 10 & 1100 & 7 & VST & HD & - & - & - & $>18$ \\
\hline T23 & & 20 & 4000 & 9 & $\mathrm{~T}$ & ST & VST & VST & 3 & $>18$ \\
\hline $\mathrm{T} 24$ & & 30 & 28500 & $>24$ & $\mathrm{~T}$ & $\mathrm{~T}$ & ST & ST & $>12$ & $>18$ \\
\hline B3 & \multirow{5}{*}{$30 \%$} & 0 & 40000 & 5 & $\mathrm{HD}$ & - & - & - & - & 9 \\
\hline T31 & & 5 & 144000 & 3.5 & HD & - & - & - & - & $>18$ \\
\hline T32 & & 10 & 250000 & 4 & VST & HD & - & - & - & $>18$ \\
\hline T33 & & 20 & 160000 & 4.5 & ST & VST & HD & - & - & $>18$ \\
\hline T34 & & 30 & 280000 & 11 & $\mathrm{~T}$ & ST & VST & VST & 3 & $>18$ \\
\hline
\end{tabular}

HD: Hard Dry $\quad$ VST: Very Slight Tacky $\quad$ ST: Slight Tacky $\quad$ T: Tacky 
FORMULATION OF NEW MODIFIED ALKYD RESINS AND THEIR APPLICATION

Table ( 13 ): Viscosity, Color, Drying time Characteristics of various HEFCA-Modified Alkyd Resins

\begin{tabular}{|c|c|c|c|c|c|c|c|c|c|c|}
\hline \multirow[b]{3}{*}{$\begin{array}{l}\text { Resin } \\
\text { Code }\end{array}$} & \multirow[b]{3}{*}{$\begin{array}{c}\text { Excess- } \\
\mathrm{OH} \\
(\%)\end{array}$} & \multirow[b]{3}{*}{$\begin{array}{c}\text { HEFCA } \\
\text { Replacement } \\
(\%)\end{array}$} & \multirow[b]{3}{*}{$\begin{array}{c}\text { Viscosity } \\
\text { (mPa.s) at solid } \\
\text { content }=70 \%\end{array}$} & \multirow{3}{*}{$\begin{array}{c}\text { Air } \\
\text { Drying } \\
\text { (HD } \\
\text { Time) } \\
\text { (hr) }\end{array}$} & \multicolumn{5}{|c|}{ Stoving Dry } & \multirow[b]{3}{*}{$\begin{array}{c}\text { Color } \\
\text { (Gardner) }\end{array}$} \\
\hline & & & & & $110 \mathrm{C}$ & & & $20 \mathrm{C}$ & & \\
\hline & & & & & $1 \mathrm{hr}$ & $2 \mathrm{hrs}$ & $1 \mathrm{hr}$ & $2 \mathrm{hrs}$ & $\begin{array}{c}\text { Extra } \\
\text { Stoving } \\
\text { Drying } \\
\text { time } \\
\text { (hrs) }\end{array}$ & \\
\hline B0 & \multirow{5}{*}{$0 \%$} & 0 & 50 & 13 & VST & HD & - & - & & 8 \\
\hline F01 & & 5 & 50 & $>24$ & VST & HD & - & - & & $>18$ \\
\hline F02 & & 10 & 75 & $>24$ & VST & HD & - & - & & $>18$ \\
\hline F03 & & 20 & 125 & $>24$ & $\mathrm{~T}$ & ST & VST & VST & $>12$ & $>18$ \\
\hline F04 & & 30 & 150 & $>24$ & $\mathrm{~T}$ & $\mathrm{~T}$ & $\mathrm{ST}$ & $\mathrm{ST}$ & $>12$ & $>18$ \\
\hline B1 & \multirow{5}{*}{$10 \%$} & 0 & 140 & 11 & VST & HD & - & - & & 8 \\
\hline F11 & & 5 & 100 & 3.5 & HD & - & - & - & & $>18$ \\
\hline F12 & & 10 & 150 & 4.0 & VST & HD & - & - & & $>18$ \\
\hline F13 & & 20 & 250 & 4.5 & $\mathrm{~T}$ & ST & VST & HD & & $>18$ \\
\hline F14 & & 30 & 325 & $>24$ & $\mathrm{~T}$ & $\mathrm{~T}$ & ST & $\mathrm{ST}$ & $>12$ & $>18$ \\
\hline B2 & \multirow{5}{*}{$20 \%$} & 0 & 450 & 7 & VST & HD & - & - & & 9 \\
\hline F21 & & 5 & 1250 & 4 & HD & - & - & - & & $>18$ \\
\hline F22 & & 10 & 1550 & 4.5 & HD & - & - & - & & $>18$ \\
\hline F23 & & 20 & 1850 & 5 & $\mathrm{~T}$ & $\mathrm{~T}$ & $\mathrm{ST}$ & $\mathrm{ST}$ & $>12$ & $>18$ \\
\hline F24 & & 30 & 2250 & $>24$ & $\mathrm{~T}$ & $\mathrm{~T}$ & ST & $\mathrm{ST}$ & $>12$ & $>18$ \\
\hline B3 & \multirow{5}{*}{$30 \%$} & 0 & 40000 & 5 & HD & - & - & - & & 9 \\
\hline F31 & & 5 & 4400 & 4 & HD & - & - & - & & $>18$ \\
\hline F32 & & 10 & 260000 & 4.5 & HD & - & - & - & & $>18$ \\
\hline F33 & & 20 & 280000 & 5 & ST & VST & VST & VST & 3 & $>18$ \\
\hline F34 & & 30 & $\begin{array}{l}25000(\text { at solid } \\
\text { content }=50 \%)\end{array}$ & 5.5 & ST & VST & VST & VST & 3 & $>18$ \\
\hline
\end{tabular}

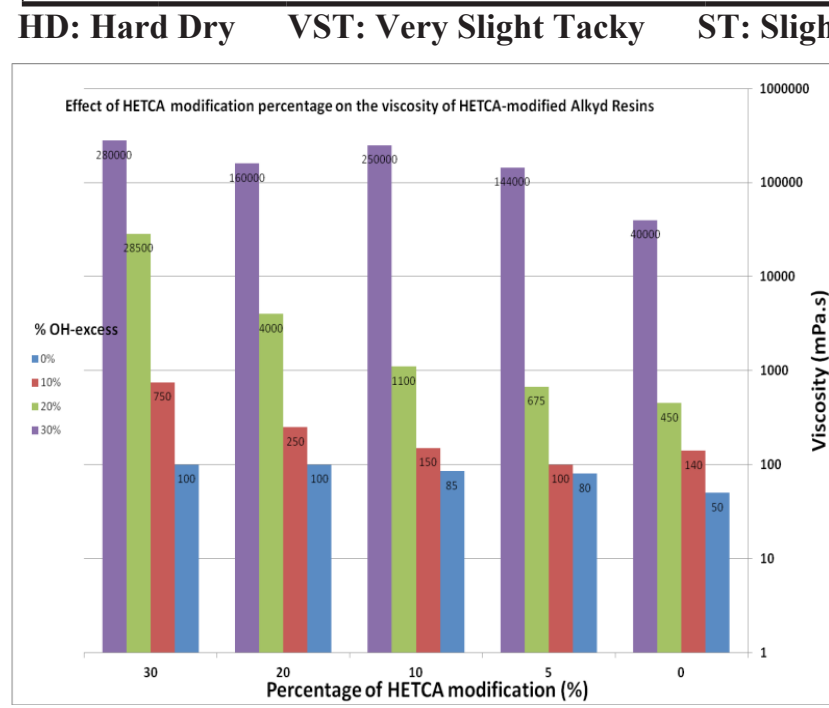

Figure: 7

The drying time increase by increasing the modifier percentage and by decreasing $\mathrm{OH}$-excess of alkyd resins. The drying time of $20 \%$ and $30 \%$ modification samples at low $\mathrm{OH}$-excess is very bad (e.g. T03, T04, F03, F04, T14, F14).

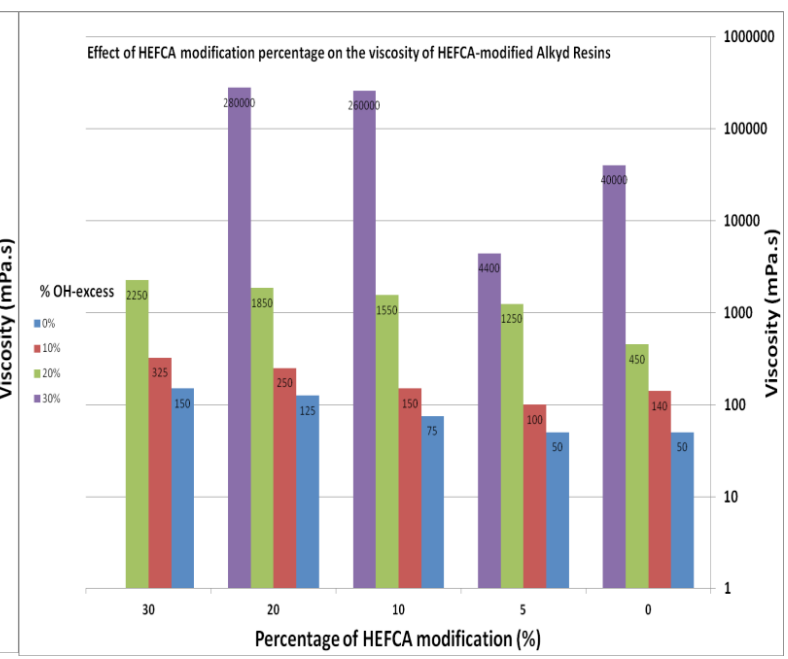

Figure: 8

The best drying time is obtained at $30 \% \mathrm{OH}$ excess resins at $5 \%$ modification (e.g. T31 and F31).

-The viscosity for various resins at constant solid content increases slightly by the increas- 
ing of the percentage of modifier, but increases dramatically with the increase of excess- $\mathrm{OH}$ percentage as shown in Figure (7 and 8)

-The color of prepared varnishes was very dark due to the presence of nitrogen atom in the modified alkyd chain.

\section{B-Water, Acid, Alkali and Solvent Resistance}

Preliminary evaluation studies are carried out to show the air drying and stoving drying films of all formulations towards water, acid, alkali and solvent resistances. The evaluation tests are conducted according to standard methods. The data are collected in tables (14 - 15).
The main conclusion drown from the water, acid, alkali and solvent resistances data for the various HETCA and HEFCA-modified alkyd is tabulated in tables $(10-11)$ indicate the following:

1. Except unmodified resins (B0, B01 and B03), all examined films show excellent water, acid and solvent resistance indicating no significant effect of the presence of the modifier within the employed experimental conditions.

2. The alkali resistance of the dried films is clearly improved upon the modification.

Table (14): Water, Acid, Alkali and solvent Resistance data for HETCA-modified Alkyd Resins

\begin{tabular}{|c|c|c|c|c|c|c|c|c|c|}
\hline \multirow{2}{*}{$\begin{array}{l}\text { Resin } \\
\text { Code }\end{array}$} & \multirow{2}{*}{$\begin{array}{c}\text { Excess- } \\
\text { OH \% }\end{array}$} & \multicolumn{2}{|c|}{$\begin{array}{c}\text { Water } \\
\text { resistance }\end{array}$} & \multicolumn{2}{|c|}{ Acid resistance } & \multicolumn{2}{|c|}{ Alkali Resistance } & \multicolumn{2}{|c|}{ Solvent Resistance } \\
\hline & & $\mathrm{A}$ & $\mathrm{S}$ & $\mathrm{A}$ & $\mathrm{S}$ & $\mathrm{A}$ & $\mathrm{S}$ & $\mathrm{A}$ & $\mathrm{S}$ \\
\hline B0 & \multirow{5}{*}{$0 \%$} & $\overline{E x}$ & $\overline{E x}$ & $\overline{E x}$ & $\overline{E x}$ & $\bar{F}$ & $P$ & $\overline{E x}$ & $\overline{E x}$ \\
\hline T01 & & Ex & Ex & Ex & Ex & Ex & Ex & Ex & Ex \\
\hline T02 & & Ex & Ex & Ex & Ex & Ex & Ex & Ex & Ex \\
\hline T03 & & Ex & Ex & Ex & Ex & Ex & Ex & Ex & Ex \\
\hline T04 & & Ex & Ex & Ex & Ex & Ex & Ex & Ex & Ex \\
\hline B1 & \multirow{5}{*}{$10 \%$} & Ex & Ex & Ex & Ex & Ex & $\mathrm{F}$ & Ex & Ex \\
\hline $\mathrm{T} 11$ & & Ex & Ex & Ex & Ex & Ex & Ex & Ex & Ex \\
\hline $\mathrm{T} 12$ & & Ex & Ex & Ex & Ex & Ex & Ex & Ex & Ex \\
\hline $\mathrm{T} 13$ & & Ex & Ex & Ex & Ex & Ex & Ex & Ex & Ex \\
\hline $\mathrm{T} 14$ & & Ex & Ex & Ex & Ex & $\mathrm{Ex}$ & Ex & Ex & $\mathrm{Ex}$ \\
\hline B2 & \multirow{5}{*}{$20 \%$} & Ex & Ex & Ex & Ex & Ex & $\mathrm{G}$ & Ex & Ex \\
\hline $\mathrm{T} 21$ & & Ex & Ex & Ex & Ex & Ex & Ex & Ex & Ex \\
\hline $\mathrm{T} 22$ & & Ex & Ex & Ex & Ex & Ex & Ex & Ex & Ex \\
\hline $\mathrm{T} 23$ & & Ex & Ex & Ex & Ex & Ex & Ex & Ex & Ex \\
\hline $\mathrm{T} 24$ & & Ex & Ex & Ex & Ex & $\mathrm{Ex}$ & $\mathrm{Ex}$ & Ex & $\mathrm{Ex}$ \\
\hline B3 & \multirow{5}{*}{$30 \%$} & $E x$ & Ex & $E x$ & Ex & $\mathrm{Ex}$ & $\mathrm{Ex}$ & Ex & $\mathrm{Ex}$ \\
\hline T31 & & Ex & Ex & Ex & Ex & Ex & Ex & Ex & Ex \\
\hline T32 & & Ex & Ex & Ex & Ex & Ex & Ex & Ex & Ex \\
\hline T33 & & Ex & Ex & Ex & Ex & Ex & Ex & Ex & Ex \\
\hline T34 & & Ex & $\mathrm{Ex}$ & $\mathrm{G}$ & Ex & $\mathrm{Ex}$ & $\mathrm{Ex}$ & Ex & $\mathrm{Ex}$ \\
\hline
\end{tabular}

Ex : Excellent (Almost no change) G : Good (Very slight change) F : Fair (Partially attacked) P : Poor (Complete film failure) S : Stoved drying film A: Air drying film 
FORMULATION OF NEW MODIFIED ALKYD RESINS AND THEIR APPLICATION

Table (15 ): Water, Acid, Alkali and solvent Resistance data for HEFCA-modified Alkyd Resins

\begin{tabular}{|c|c|c|c|c|c|c|c|c|c|}
\hline \multirow{2}{*}{$\begin{array}{l}\text { Resin } \\
\text { Code }\end{array}$} & \multirow{2}{*}{$\begin{array}{c}\text { Excess- } \\
\mathrm{OH} \% \\
\end{array}$} & \multicolumn{2}{|c|}{ Water resistance } & \multicolumn{2}{|c|}{ Acid resistance } & \multicolumn{2}{|c|}{ Alkali Resistance } & \multicolumn{2}{|c|}{ Solvent Resistance } \\
\hline & & $\mathrm{A}$ & $\mathrm{S}$ & $\mathrm{A}$ & $\mathrm{S}$ & A & $\mathrm{S}$ & A & $\mathrm{S}$ \\
\hline B0 & \multirow{5}{*}{$0 \%$} & Ex & Ex & Ex & Ex & $\mathrm{F}$ & $\mathrm{P}$ & Ex & Ex \\
\hline F01 & & Ex & Ex & Ex & Ex & Ex & Ex & Ex & Ex \\
\hline F02 & & Ex & Ex & Ex & Ex & Ex & Ex & Ex & Ex \\
\hline F03 & & Ex & Ex & Ex & Ex & Ex & Ex & Ex & Ex \\
\hline F04 & & Ex & Ex & Ex & Ex & Ex & Ex & Ex & Ex \\
\hline B1 & \multirow{5}{*}{$10 \%$} & $\mathrm{Ex}$ & $\mathrm{Ex}$ & Ex & Ex & Ex & $\mathrm{F}$ & Ex & Ex \\
\hline F11 & & Ex & Ex & Ex & Ex & Ex & Ex & Ex & Ex \\
\hline F12 & & Ex & Ex & Ex & Ex & Ex & Ex & Ex & Ex \\
\hline F13 & & Ex & Ex & Ex & Ex & Ex & Ex & Ex & Ex \\
\hline F14 & & Ex & Ex & Ex & Ex & Ex & Ex & Ex & Ex \\
\hline B2 & \multirow{5}{*}{$20 \%$} & $\mathrm{Ex}$ & $\mathrm{Ex}$ & $\mathrm{Ex}$ & Ex & Ex & $\mathrm{G}$ & Ex & Ex \\
\hline F21 & & Ex & Ex & Ex & Ex & Ex & Ex & Ex & Ex \\
\hline F22 & & Ex & Ex & Ex & Ex & Ex & Ex & Ex & Ex \\
\hline F23 & & Ex & Ex & Ex & Ex & Ex & Ex & Ex & Ex \\
\hline F24 & & Ex & Ex & Ex & Ex & Ex & Ex & Ex & Ex \\
\hline B3 & \multirow{5}{*}{$30 \%$} & $\mathrm{Ex}$ & $\mathrm{Ex}$ & Ex & Ex & Ex & Ex & Ex & Ex \\
\hline F31 & & Ex & Ex & Ex & Ex & Ex & Ex & Ex & Ex \\
\hline F32 & & Ex & Ex & Ex & Ex & Ex & Ex & Ex & Ex \\
\hline F33 & & Ex & Ex & Ex & Ex & Ex & Ex & Ex & Ex \\
\hline F34 & & Ex & Ex & $\mathrm{G}$ & Ex & Ex & Ex & Ex & Ex \\
\hline
\end{tabular}

Ex : Excellent (Almost no change)

F : Fair (Partially attacked)

A: Air drying film

\section{Extensive Evaluation Studies:}

Attention the directed towards more extensive studies to indicate how much the films can withstand the various attacks in terms of immersion periods. The extensive evaluation studies for water resistance, acid resistance and solvent resistance are given in table (16). The tests are carried out on the actual coating films (air dried and stoving dry) for 30 days immersion period and the films are examined for any defects.

Additionally, the extensive evaluation stud-
G : Good (Very slight change)

P : Poor (Complete film failure)

$S$ : Stoved drying film

ies for alkali resistance are shown in tables (17) which are carried out on the actual coating films (air dried and stoving dry) for 10 days immersion period and the films are examined for any defects.

In general, water, acid and solvent resistance for all resins are good after 30 days for both air and stoving films.

The alkali resistance of resins generally improved by increasing the modification and excess-OH\% of the prepared alkyd resins. 
M. M. ELSHAHAWI, et al.

Table (16):Water, Acid, Alkali and solvent Resistance data for modified Alkyd Resins after 30 days

\begin{tabular}{|c|c|c|c|c|c|c|c|c|c|c|c|c|c|c|c|}
\hline \multirow{2}{*}{$\begin{array}{l}\text { Resin } \\
\text { Code }\end{array}$} & \multirow{2}{*}{$\begin{array}{l}\text { Excess- } \\
\mathrm{OH} \%\end{array}$} & \multicolumn{2}{|c|}{$\begin{array}{c}\text { Water } \\
\text { resistance }\end{array}$} & \multicolumn{2}{|c|}{$\begin{array}{c}\text { Acid } \\
\text { resistance }\end{array}$} & \multicolumn{2}{|c|}{$\begin{array}{c}\text { Solvent } \\
\text { Resistance }\end{array}$} & \multirow{2}{*}{$\begin{array}{l}\text { Resin } \\
\text { Code }\end{array}$} & \multirow{2}{*}{$\begin{array}{c}\text { Excess- } \\
\mathrm{OH} \%\end{array}$} & \multicolumn{2}{|c|}{$\begin{array}{c}\text { Water } \\
\text { resistance }\end{array}$} & \multicolumn{2}{|c|}{$\begin{array}{c}\text { Acid } \\
\text { resistance }\end{array}$} & \multicolumn{2}{|c|}{$\begin{array}{c}\text { Solvent } \\
\text { Resistance }\end{array}$} \\
\hline & & A & $\mathrm{S}$ & A & $\mathrm{S}$ & A & $\mathrm{S}$ & & & A & $\mathrm{S}$ & A & $\mathrm{S}$ & A & $\mathrm{S}$ \\
\hline B0 & \multirow{5}{*}{$0 \%$} & Ex & Ex & Ex & Ex & Ex & Ex & B0 & \multirow{5}{*}{$0 \%$} & Ex & Ex & Ex & Ex & Ex & Ex \\
\hline T01 & & Ex & Ex & Ex & Ex & Ex & Ex & F01 & & Ex & Ex & Ex & Ex & Ex & Ex \\
\hline T02 & & Ex & Ex & Ex & Ex & Ex & Ex & F02 & & Ex & Ex & Ex & Ex & Ex & Ex \\
\hline $\mathrm{T} 03$ & & Ex & Ex & Ex & Ex & Ex & Ex & F03 & & Ex & Ex & Ex & Ex & Ex & Ex \\
\hline T04 & & Ex & Ex & Ex & Ex & Ex & Ex & F04 & & Ex & Ex & Ex & Ex & Ex & Ex \\
\hline B1 & \multirow{5}{*}{$10 \%$} & $\overline{E x}$ & Ex & $\overline{E x}$ & $\overline{E x}$ & $\overline{E x}$ & Ex & $\bar{B} 1$ & \multirow{5}{*}{$10 \%$} & $\overline{E x}$ & $\overline{E x}$ & $\overline{E x}$ & $\overline{E x}$ & Ex & $\overline{E x}$ \\
\hline T11 & & Ex & Ex & Ex & Ex & Ex & Ex & F11 & & Ex & Ex & Ex & Ex & Ex & Ex \\
\hline $\mathrm{T} 12$ & & Ex & Ex & Ex & Ex & Ex & Ex & F12 & & Ex & Ex & Ex & Ex & Ex & Ex \\
\hline T13 & & Ex & Ex & Ex & Ex & Ex & Ex & F13 & & Ex & Ex & Ex & Ex & Ex & Ex \\
\hline T14 & & Ex & Ex & Ex & Ex & Ex & Ex & F14 & & Ex & Ex & Ex & Ex & Ex & Ex \\
\hline B2 & \multirow{5}{*}{$20 \%$} & $E x$ & $\mathrm{Ex}$ & Ex & $E x$ & Ex & $E x$ & B2 & \multirow{5}{*}{$20 \%$} & $E x$ & $E x$ & $E x$ & Ex & $E x$ & Ex \\
\hline $\mathrm{T} 21$ & & Ex & Ex & Ex & Ex & Ex & Ex & F21 & & Ex & Ex & Ex & Ex & Ex & Ex \\
\hline $\mathrm{T} 22$ & & Ex & Ex & Ex & Ex & Ex & Ex & F22 & & $E x$ & Ex & Ex & Ex & Ex & Ex \\
\hline $\mathrm{T} 23$ & & Ex & Ex & Ex & Ex & Ex & Ex & F23 & & Ex & Ex & Ex & Ex & Ex & Ex \\
\hline $\mathrm{T} 24$ & & Ex & Ex & Ex & Ex & Ex & Ex & F24 & & Ex & Ex & Ex & Ex & Ex & Ex \\
\hline B3 & \multirow{5}{*}{$30 \%$} & $\overline{E x}$ & Ex & $\overline{E x}$ & $\overline{E x}$ & $\overline{E x}$ & Ex & $\overline{\text { B3 }}$ & \multirow{5}{*}{$30 \%$} & $\overline{E x}$ & Ex & $\overline{E x}$ & $\overline{E x}$ & $\overline{E x}$ & $\overline{E x}$ \\
\hline T31 & & Ex & Ex & Ex & Ex & Ex & Ex & F31 & & Ex & Ex & Ex & Ex & Ex & Ex \\
\hline T32 & & Ex & Ex & Ex & Ex & Ex & Ex & F32 & & Ex & Ex & Ex & Ex & Ex & Ex \\
\hline T33 & & Ex & Ex & Ex & Ex & Ex & Ex & F33 & & Ex & Ex & Ex & Ex & Ex & Ex \\
\hline T34 & & Ex & Ex & $\mathrm{G}$ & Ex & Ex & Ex & F34 & & Ex & Ex & $\mathrm{G}$ & Ex & Ex & Ex \\
\hline
\end{tabular}

Ex : Excellent (Almost no change)

G : Good (Very slight change) F : Fair

(Partially attacked) $\quad \mathbf{P}$ : Poor (Complete film failure) A: Air drying film $\mathbf{S}:$ Stoved drying film

Table (17): Alkali and solvent Resistance data for modified Alkyd Resins after 10 days

\begin{tabular}{|c|c|c|c|c|c|c|c|}
\hline \multirow[t]{2}{*}{ Resin Code } & \multirow[t]{2}{*}{ Excess-OH \% } & \multicolumn{2}{|c|}{$\begin{array}{c}\text { Alkali resistance (after } \\
10 \text { days) }\end{array}$} & \multirow[t]{2}{*}{ Resin Code } & \multirow{2}{*}{$\begin{array}{c}\text { Excess- } \\
\text { OH \% }\end{array}$} & \multicolumn{2}{|c|}{$\begin{array}{c}\text { Alkali resistance (after } \\
10 \text { days) }\end{array}$} \\
\hline & & A & $\mathrm{S}$ & & & A & $\mathrm{S}$ \\
\hline B0 & \multirow{5}{*}{$0 \%$} & $P$ & $P$ & B0 & \multirow{5}{*}{$0 \%$} & $P$ & $P$ \\
\hline T01 & & $P$ & Ex & F01 & & $P$ & Ex \\
\hline T02 & & $P$ & Ex & F02 & & $P$ & Ex \\
\hline T03 & & $P$ & Ex & F03 & & $P$ & $\mathrm{P}$ \\
\hline T04 & & G & G & F04 & & $P$ & $P$ \\
\hline$\overline{\mathrm{B} 1}$ & \multirow{5}{*}{$10 \%$} & $\bar{P}$ & $\bar{P}$ & $\overline{\mathrm{B} 1}$ & \multirow{5}{*}{$10 \%$} & $\bar{P}$ & $\bar{P}$ \\
\hline T11 & & $\mathrm{P}$ & Ex & F11 & & $\mathrm{F}$ & Ex \\
\hline T12 & & $\mathrm{F}$ & Ex & F12 & & G & Ex \\
\hline T13 & & Ex & Ex & F13 & & Ex & Ex \\
\hline $\mathrm{T} 14$ & & Ex & Ex & F14 & & Ex & $\mathrm{F}$ \\
\hline B2 & \multirow{5}{*}{$20 \%$} & $\bar{F}$ & $P$ & B2 & \multirow{5}{*}{$20 \%$} & $\bar{F}$ & $\bar{P}$ \\
\hline $\mathrm{T} 21$ & & Ex & Ex & F21 & & Ex & Ex \\
\hline $\mathrm{T} 22$ & & Ex & Ex & F22 & & Ex & Ex \\
\hline T23 & & Ex & Ex & F23 & & Ex & G \\
\hline $\mathrm{T} 24$ & & Ex & Ex & F24 & & Ex & $\mathrm{P}$ \\
\hline B3 & \multirow{5}{*}{$30 \%$} & Ex & $\overline{\mathrm{G}}$ & B3 & \multirow{5}{*}{$30 \%$} & $\overline{E x}$ & $\bar{G}$ \\
\hline T31 & & Ex & Ex & F31 & & Ex & Ex \\
\hline T32 & & Ex & $\mathrm{P}$ & F32 & & Ex & Ex \\
\hline T33 & & Ex & Ex & F33 & & Ex & $\mathrm{F}$ \\
\hline $\mathrm{T} 34$ & & Ex & Ex & F34 & & Ex & $\mathrm{P}$ \\
\hline
\end{tabular}

Ex : Excellent (Almost no change) G : Good (Very slight change) $\mathrm{F}$ : Fair (Partially attacked) P : Poor (Complete film failure) A: Air drying film $\quad$ S : Stoved drying film

\section{C- Mechanical properties of dried films:}

The dry film characteristics of various modified resin films are carried out according to standard methods and well-known techniques and the results are tabulated in tables $(19,20$,
21 and 22). These tables show some mechanical properties such as gloss, hardness, scratch resistance and adhesion of both varnishes and primerscontaining modified alkyd resins. The formulation of primer will be as shown in the following table: 
Table (18): Zinc Phosphate Primer formula

\begin{tabular}{|c|c|c|}
\hline serial & Component & $\%$ \\
\hline 1 & Resin/modified resin & 30 \\
\hline 2 & Bentone38 & 1 \\
\hline 3 & Ethanol & 0.3 \\
\hline 4 & Wetting \& dispersing Agent & 0.3 \\
\hline 5 & $\mathrm{TiO}_{2}$ & 10 \\
\hline 6 & Zinc phosphate & 5 \\
\hline 7 & $\mathrm{CaCO}_{3}$ & 24 \\
\hline 8 & Talc & 5 \\
\hline 9 & Xylene & 22.21 \\
\hline 10 & Calcium Octoate $(10 \%)$ & 1 \\
\hline 11 & Cobalt octoate (10\%) & 0.43 \\
\hline 12 & Zirconium Octoate (12\%) & 0.63 \\
\hline 13 & Ex skin 2 & 0.13 \\
\hline \multicolumn{2}{|l|}{ Total } & 100 \\
\hline
\end{tabular}

\begin{tabular}{|c|c|c|c|c|c|c|c|c|c|c|c|c|c|}
\hline \multirow{2}{*}{ Resin Code } & \multirow{2}{*}{$\begin{array}{l}\text { Excess- } \\
\text { OH \% }\end{array}$} & \multicolumn{3}{|c|}{ Film Thickness $(\mu)$} & \multicolumn{3}{|c|}{ "Gloss at $20^{0}$} & \multicolumn{3}{|c|}{ Flexibility at $3 \mathrm{~mm}$} & \multicolumn{3}{|c|}{ Adhesion } \\
\hline & & $\mathbf{A}$ & $\mathbf{S}$ & $\mathbf{P}$ & $\mathbf{A}$ & $\mathbf{S}$ & $\mathbf{P}$ & $\mathbf{A}$ & $\mathbf{S}$ & $\mathbf{P}$ & $\mathbf{A}$ & $\mathbf{S}$ & $\mathbf{P}$ \\
\hline$\overline{\text { B0 }}$ & \multirow{5}{*}{$0 \%$} & 42 & 27 & 75 & 76 & 100 & $\overline{4}$ & $\overline{E x}$ & $\overline{E x}$ & $\mathrm{Ex}$ & $\overline{5 B}$ & $5 \mathrm{~B}$ & $\overline{5 \mathrm{~B}}$ \\
\hline T01 & & 25 & 20 & 37 & 81 & 93 & 4 & Ex & Ex & Ex & $5 \mathrm{~B}$ & $5 \mathrm{~B}$ & $5 \mathrm{~B}$ \\
\hline T02 & & 25 & 25 & 57 & 82 & 85 & 5 & Ex & Ex & Ex & $5 \mathrm{~B}$ & $5 \mathrm{~B}$ & $5 \mathrm{~B}$ \\
\hline T03 & & 35 & 20 & 45 & 85 & 93 & 11 & Ex & Ex & $\mathrm{Ex}$ & $5 \mathrm{~B}$ & $5 \mathrm{~B}$ & $5 \mathrm{~B}$ \\
\hline T04 & & 30 & 25 & 36 & 90 & 95 & 7 & Ex & Ex & Ex & 5B & $5 \mathrm{~B}$ & $5 \mathrm{~B}$ \\
\hline B1 & \multirow{5}{*}{$10 \%$} & 25 & 25 & 42 & 77 & 96 & 10 & Ex & Ex & Ex & $5 \mathrm{~B}$ & $5 \mathrm{~B}$ & 4B \\
\hline T11 & & 40 & 25 & 43 & 79 & 90 & 5 & Ex & Ex & Ex & 4B & $5 \mathrm{~B}$ & $5 \mathrm{~B}$ \\
\hline T12 & & 40 & 25 & 75 & 81 & 88 & 7 & Ex & Ex & Ex & 5B & $5 \mathrm{~B}$ & $5 \mathrm{~B}$ \\
\hline T13 & & 25 & 25 & 48 & 82 & 84 & 21 & Ex & Ex & Ex & 5B & $5 \mathrm{~B}$ & $4 \mathrm{~B}$ \\
\hline T14 & & 25 & 30 & 75 & 90 & 70 & 42 & Ex & Ex & Ex & 5B & $5 \mathrm{~B}$ & $5 \mathrm{~B}$ \\
\hline B2 & \multirow{5}{*}{$20 \%$} & 35 & 30 & 61 & 83 & 98 & 4 & Ex & Ex & \begin{tabular}{|l} 
Ex at $5 \mathrm{~mm}$ \\
\end{tabular} & $5 \mathrm{~B}$ & 5B & $5 \mathrm{~B}$ \\
\hline T21 & & 50 & 50 & 60 & 79 & 95 & 6 & Ex & Ex & Ex & $2 \mathrm{~B}$ & $5 \mathrm{~B}$ & $5 \mathrm{~B}$ \\
\hline T22 & & 70 & 50 & 65 & 83 & 67 & 24 & Ex & Ex & Ex & $5 \mathrm{~B}$ & $5 \mathrm{~B}$ & $5 \mathrm{~B}$ \\
\hline T23 & & 55 & 50 & 50 & 81 & 89 & 10 & Ex & Ex & Ex & $2 \mathrm{~B}$ & $5 \mathrm{~B}$ & $5 \mathrm{~B}$ \\
\hline T24 & & 60 & 40 & 50 & 93 & 89 & 25 & Ex & Ex & Ex & $5 \mathrm{~B}$ & $5 \mathrm{~B}$ & $4 \mathrm{~B}$ \\
\hline B3 & \multirow{5}{*}{$30 \%$} & 35 & 20 & 56 & 96 & 99 & 11 & Ex & Ex & Ex & $0 \mathrm{~B}$ & $5 \mathrm{~B}$ & $5 \mathrm{~B}$ \\
\hline T31 & & 40 & 45 & 63 & 89 & 97 & 9 & Ex & Ex & Ex & 0B & $5 \mathrm{~B}$ & $5 \mathrm{~B}$ \\
\hline T32 & & 45 & 30 & 70 & 92 & 89 & 35 & Ex & Ex & Ex at $10 \mathrm{~mm}$ & 0B & $5 \mathrm{~B}$ & $4 \mathrm{~B}$ \\
\hline T33 & & 35 & 25 & 60 & 84 & 76 & 27 & Ex & Ex & Ex & 0B & $5 \mathrm{~B}$ & $4 \mathrm{~B}$ \\
\hline T34 & & 30 & 21 & 70 & 84 & 92 & 15 & Ex & Ex & Ex & $0 \mathrm{~B}$ & $5 \mathrm{~B}$ & $2 \mathrm{~B}$ \\
\hline
\end{tabular}

0B : Removed area is greater than $65 \%, 1 \mathrm{~B}: 35-65 \%$ removed area, $2 \mathrm{~B}: 15-35 \%$ removed area, 3B : $5-15 \%$ removed area, $4 \mathrm{~B}$ : Less than 5\%, 5B: $0 \%$ removed area

Table (19): Primer and Varnish Characteristic of Various HETCA-modified Alkyd Resins

\begin{tabular}{|c|c|c|c|c|c|c|c|}
\hline \multirow{2}{*}{ Resin Code } & \multirow{2}{*}{$\begin{array}{r}\text { Excess-OH } \\
\%\end{array}$} & \multicolumn{3}{|c|}{ Impact (1 Kg ) Excellent at (cm) } & \multicolumn{3}{|c|}{$\begin{array}{r}\text { Scratch Hardness (Pencil } \\
\text { Hardness) }\end{array}$} \\
\hline & & $\mathbf{A}$ & $\mathbf{S}$ & $\mathbf{P}$ & $\mathbf{A}$ & $\mathbf{S}$ & $\mathbf{P}$ \\
\hline B0 & \multirow{5}{*}{$0 \%$} & 100 & 100 & 100 & $2 \mathrm{~B}$ & $8 \mathrm{H}$ & $9 \mathrm{H}$ \\
\hline T01 & & 100 & 100 & 100 & $\mathrm{~B}$ & $6 \mathrm{H}$ & $9 \mathrm{H}$ \\
\hline T02 & & 100 & 100 & 100 & $5 \mathrm{~B}$ & $3 \mathrm{H}$ & $9 \mathrm{H}$ \\
\hline T03 & & 100 & 100 & 100 & $6 \mathrm{~B}$ & $4 \mathrm{H}$ & $8 \mathrm{H}$ \\
\hline T04 & & 100 & 100 & 100 & $6 \mathrm{~B}$ & $6 \mathrm{H}$ & 3B \\
\hline B1 & \multirow{5}{*}{$10 \%$} & 100 & 100 & 100 & $2 \mathrm{~B}$ & $6 \mathrm{H}$ & $\overline{4 \mathrm{H}}$ \\
\hline T11 & & 100 & 100 & 100 & $2 \mathrm{~B}$ & $8 \mathrm{H}$ & $9 \mathrm{H}$ \\
\hline T12 & & 100 & 100 & 100 & $2 \mathrm{~B}$ & $6 \mathrm{H}$ & $6 \mathrm{H}$ \\
\hline T13 & & 100 & 100 & 100 & $2 \mathrm{~B}$ & $6 \mathrm{H}$ & $5 \mathrm{H}$ \\
\hline T14 & & 100 & 100 & 100 & $5 B$ & $6 \mathrm{H}$ & $4 \mathrm{H}$ \\
\hline B2 & \multirow{5}{*}{$20 \%$} & 100 & 100 & 100 & $3 \mathrm{~B}$ & $7 \mathrm{H}$ & $\overline{9 \mathrm{H}}$ \\
\hline T21 & & 90 & 100 & 100 & $2 \mathrm{~B}$ & $\mathrm{HB}$ & $9 \mathrm{H}$ \\
\hline T22 & & 60 & 100 & 100 & $2 \mathrm{~B}$ & $\mathrm{HB}$ & $3 \mathrm{~B}$ \\
\hline T23 & & 100 & 100 & 100 & $2 \mathrm{~B}$ & $\mathrm{HB}$ & $5 \mathrm{H}$ \\
\hline T24 & & 90 & 100 & 100 & $2 \mathrm{~B}$ & $\mathrm{HB}$ & $4 \mathrm{H}$ \\
\hline B3 & \multirow{5}{*}{$30 \%$} & 90 & 100 & 100 & $7 \mathrm{H}$ & $9 \mathrm{H}$ & $7 \mathrm{HH}$ \\
\hline T31 & & 55 & 100 & 100 & $7 \mathrm{H}$ & $2 \mathrm{H}$ & $6 \mathrm{H}$ \\
\hline T32 & & 90 & 100 & 100 & $5 \mathrm{H}$ & $2 \mathrm{H}$ & $6 \mathrm{H}$ \\
\hline T33 & & 80 & 100 & 100 & $\mathrm{H}$ & $\mathrm{F}$ & $3 \mathrm{H}$ \\
\hline T34 & & 75 & 100 & 100 & $\mathrm{H}$ & $\mathrm{F}$ & $3 \mathrm{H}$ \\
\hline
\end{tabular}

$6 \mathrm{~B}<\mathbf{5 B}<\mathbf{4 B}<\mathbf{3 B}<\mathbf{2 B}<\mathrm{B}<\mathrm{HB}<\mathrm{F}<\mathrm{H}<\mathbf{2 H}<\mathbf{3 H}<\mathbf{4 H}<\mathbf{5 H}<\mathbf{6 H}<\mathbf{7 H}<\mathbf{8 H}<\mathbf{9 H}$

Softer

$\rightarrow$ Harder

Table (20) Impact and Pencil hardness of HETCA-modified alkyd resin 


\begin{tabular}{|c|c|c|c|c|c|c|c|c|c|c|c|c|c|}
\hline \multirow{2}{*}{$\begin{array}{r}\text { Resin } \\
\text { Code }\end{array}$} & \multirow{2}{*}{$\begin{array}{r}\text { Excess-OH } \\
\%\end{array}$} & \multicolumn{3}{|c|}{ Film Thickness $(\mu)$} & \multicolumn{3}{|c|}{ Gloss at $60 \square$} & \multicolumn{3}{|c|}{ Flexibility at $3 \mathrm{~mm}$} & \multicolumn{3}{|c|}{ Adhesion } \\
\hline & & $\mathbf{A}$ & $\mathbf{S}$ & $\mathbf{P}$ & $\mathbf{A}$ & $\mathbf{S}$ & $\mathbf{P}$ & $\mathbf{A}$ & $\mathbf{S}$ & $\mathbf{P}$ & $\mathbf{A}$ & $\mathbf{S}$ & $\mathbf{P}$ \\
\hline B0 & \multirow{5}{*}{ 0\% } & 42 & 27 & 75 & 76 & 100 & 4 & Ex & Ex & Ex & $5 \mathrm{~B}$ & $5 \mathrm{~B}$ & $5 \mathrm{~B}$ \\
\hline F01 & & 35 & 25 & 66 & 79 & 80 & 14 & Ex & Ex & Ex & 5B & 5B & $5 \mathrm{~B}$ \\
\hline F02 & & 40 & 30 & 40 & 79 & 92 & 9 & Ex & Ex & Ex & $5 \mathrm{~B}$ & $5 \mathrm{~B}$ & $5 \mathrm{~B}$ \\
\hline F03 & & 35 & 25 & 39 & 75 & 91 & 14 & Ex & Ex & Ex & $5 \mathrm{~B}$ & $5 \mathrm{~B}$ & 4B \\
\hline F04 & & 30 & 20 & 50 & 79 & 80 & 6 & Ex & Ex & Ex & 5B & $5 \mathrm{~B}$ & 4B \\
\hline B1 & \multirow{5}{*}{$10 \%$} & 25 & 25 & 77 & 77 & 96 & 10 & Ex & Ex & $\overline{E x}$ & 5B & 5B & $4 \mathrm{~B}$ \\
\hline F11 & & 45 & 35 & 40 & 77 & 73 & 13 & Ex & Ex & Ex & $5 \mathrm{~B}$ & 5B & 5B \\
\hline F12 & & 50 & 40 & 34 & 77 & 75 & 6 & Ex & Ex & Ex & $5 \mathrm{~B}$ & $5 \mathrm{~B}$ & 4B \\
\hline F13 & & 40 & 35 & 66 & 79 & 84 & 7 & Ex & Ex & Ex & $5 \mathrm{~B}$ & $5 \mathrm{~B}$ & $5 \mathrm{~B}$ \\
\hline F14 & & 40 & 45 & 60 & 76 & 91 & 9 & Ex & Ex & Ex & 5B & $5 \mathrm{~B}$ & 4B \\
\hline B2 & \multirow{5}{*}{$20 \%$} & 35 & 30 & 61 & 83 & 98 & 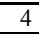 & Ex & Ex & Ex & $5 \mathrm{~B}$ & 5B & 5B \\
\hline F21 & & 65 & 50 & 44 & 82 & 72 & 12 & Ex & Ex & Ex & 0B & $5 \mathrm{~B}$ & $5 \mathrm{~B}$ \\
\hline F22 & & 45 & 62 & 60 & 77 & 87 & 7 & Ex & Ex & Ex & $0 \mathrm{~B}$ & $5 \mathrm{~B}$ & $5 \mathrm{~B}$ \\
\hline F23 & & 30 & 50 & 90 & 80 & 71 & 10 & Ex & Ex & Ex & $2 \mathrm{~B}$ & $5 \mathrm{~B}$ & $5 \mathrm{~B}$ \\
\hline F24 & & 70 & 26 & 70 & 82 & 79 & 11 & Ex & Ex & Ex & 0B & $5 \mathrm{~B}$ & $5 B$ \\
\hline B3 & \multirow{5}{*}{$30 \%$} & 35 & 20 & 56 & 96 & 99 & 11 & Ex & Ex & Ex & $0 \mathrm{~B}$ & 5B & 5B \\
\hline F31 & & 30 & 20 & 75 & 91 & 66 & 6 & Ex & Ex & Ex & 0B & 5B & $5 \mathrm{~B}$ \\
\hline F32 & & 43 & 30 & 70 & 86 & 66 & 7 & Ex & Ex & Ex & $0 \mathrm{~B}$ & 5B & 5B \\
\hline F33 & & 25 & 27 & 100 & 84 & 36 & 6 & Ex & Ex & Ex & 0B & 5B & 4B \\
\hline F34 & & 15 & 13 & 56 & 77 & 77 & 3 & Ex & Ex & Ex & $2 \mathrm{~B}$ & 5B & $5 \mathrm{~B}$ \\
\hline
\end{tabular}

Ex: Excellent 0B : Removed area is greater than 65\%, 1B : 35 - 65\% removed area, 2B : $15-35 \%$ removed area, 3B : 5 - 15\% removed area,

Table (21): Primer and Varnish Characteristic of Various HEFCA-modified Alkyd Resins

\begin{tabular}{|c|c|c|c|c|c|c|c|}
\hline \multirow{2}{*}{ Resin Code } & \multirow{2}{*}{$\begin{array}{l}\text { Excess-OH } \\
\%\end{array}$} & \multicolumn{3}{|c|}{ Impact (1 Kg ) Excellent at (cm) } & \multicolumn{3}{|c|}{$\begin{array}{l}\text { Scratch Hardness (Pencil } \\
\text { Hardness) }\end{array}$} \\
\hline & & $\mathbf{A}$ & $\mathbf{S}$ & $\mathbf{P}$ & $\mathbf{A}$ & $\mathbf{S}$ & $\mathbf{P}$ \\
\hline B0 & \multirow{5}{*}{$0 \%$} & 100 & 100 & 100 & $2 \mathrm{~B}$ & $8 \mathrm{H}$ & $9 \mathrm{H}$ \\
\hline F01 & & 100 & 100 & 100 & $\mathrm{~B}$ & $6 \mathrm{H}$ & $9 \mathrm{H}$ \\
\hline F02 & & 100 & 100 & 100 & $2 \mathrm{~B}$ & $7 \mathrm{H}$ & $4 \mathrm{H}$ \\
\hline F03 & & 100 & 100 & 100 & 4B & $7 \mathrm{H}$ & $2 \mathrm{~B}$ \\
\hline F04 & & 100 & 100 & 100 & $5 \mathrm{~B}$ & $7 \mathrm{H}$ & $\mathrm{F}$ \\
\hline B1 & \multirow{5}{*}{$10 \%$} & 100 & 100 & 100 & $2 \mathrm{~B}$ & $6 \mathrm{H}$ & $\overline{44 \mathrm{H}}$ \\
\hline F11 & & 100 & 100 & 100 & $\mathrm{~F}$ & $7 \mathrm{H}$ & $6 \mathrm{H}$ \\
\hline F12 & & 100 & 100 & 100 & B & $3 \mathrm{H}$ & $4 \mathrm{H}$ \\
\hline F13 & & 100 & 100 & 100 & B & $3 \mathrm{H}$ & $9 \mathrm{H}$ \\
\hline F14 & & 100 & 100 & 100 & 4B & $2 \mathrm{H}$ & $6 \mathrm{H}$ \\
\hline$\overline{\mathrm{B2}}$ & \multirow{5}{*}{$20 \%$} & 100 & 100 & 100 & $3 \mathrm{~B}$ & $\overline{7 \mathrm{H}}$ & $\overline{\overline{9 H}}$ \\
\hline F21 & & 100 & 100 & 100 & $\mathrm{HB}$ & $6 \mathrm{H}$ & $3 \mathrm{H}$ \\
\hline F22 & & 100 & 100 & 100 & HB & $6 \mathrm{H}$ & $2 \mathrm{H}$ \\
\hline F23 & & 100 & 100 & 100 & $\mathrm{H}$ & $6 \mathrm{H}$ & $2 \mathrm{H}$ \\
\hline F24 & & 100 & 100 & 100 & $\mathrm{H}$ & $5 \mathrm{H}$ & $9 \mathrm{H}$ \\
\hline B3 & \multirow{5}{*}{$30 \%$} & 90 & 100 & 100 & $7 \mathrm{H}$ & $9 \mathrm{H}$ & $7 \mathrm{H}$ \\
\hline F31 & & 35 & 100 & 100 & $6 \mathrm{H}$ & $6 \mathrm{H}$ & $6 \mathrm{H}$ \\
\hline F32 & & 40 & 100 & 100 & $6 \mathrm{H}$ & $4 \mathrm{H}$ & $6 \mathrm{H}$ \\
\hline F33 & & 70 & 100 & 100 & $6 \mathrm{H}$ & $3 \mathrm{H}$ & $7 \mathrm{H}$ \\
\hline F34 & & 80 & 100 & 100 & $\mathrm{H}$ & $3 \mathrm{H}$ & $5 \mathrm{H}$ \\
\hline
\end{tabular}

$6 \mathrm{~B}<5 \mathrm{~B}<4 \mathrm{~B}<3 \mathrm{~B}<2 \mathrm{~B}<\mathrm{B}<\mathrm{HB}<\mathrm{F}<\mathrm{H}<2 \mathrm{H}<3 \mathrm{H}<\mathbf{4 H}<5 \mathrm{H}<6 \mathrm{H}<\mathbf{H}<\mathbf{8 H}<9 \mathrm{H}$

Softer

$\rightarrow$ Harder

Table ( 22 ) Impact and Pencil hardness of HEFCA-modified alkyd resins

The evaluation the corrosion resistance of prepared modified alkyd resins and corrosion inhibitors are carried out by dipping in 5\% NaCL solution for 240 hours. The results are shown in the following tables: 
Table (23)

Evaluation the corrosion resistance of modified alkyd resins

\begin{tabular}{|c|c|c|c|c|c|}
\hline \multirow{2}{*}{ Resin Code } & \multicolumn{2}{|l|}{ Blistering } & \multirow{2}{*}{ Resin Code } & \multicolumn{2}{|l|}{ Blistering } \\
\hline & Size & Frequency & & Size & Frequency \\
\hline B0 & 10 & $\bar{F}$ & $\overline{\text { B0 }}$ & 10 & $\overline{\mathrm{F}}$ \\
\hline T01 & 10 & $\mathrm{~F}$ & F01 & 10 & $\mathrm{~F}$ \\
\hline T02 & 10 & $\mathrm{~F}$ & F02 & 10 & $\mathrm{~F}$ \\
\hline T03 & 10 & F & F03 & 10 & $\mathrm{~F}$ \\
\hline T04 & 10 & $\mathrm{~F}$ & F04 & 10 & $\mathrm{~F}$ \\
\hline B1 & 10 & $\mathrm{~F}$ & B1 & 10 & $\mathrm{~F}$ \\
\hline T11 & 10 & $\mathrm{~F}$ & F11 & 10 & $\mathrm{~F}$ \\
\hline T12 & 10 & $\mathrm{~F}$ & F12 & 10 & $\mathrm{~F}$ \\
\hline T13 & 10 & $\mathrm{~F}$ & F13 & 10 & $\mathrm{~F}$ \\
\hline T14 & 10 & $\mathrm{~F}$ & F14 & 10 & $\mathrm{~F}$ \\
\hline B2 & 10 & $\mathrm{~F}$ & B2 & 10 & $\mathrm{~F}$ \\
\hline T21 & 10 & $\mathrm{~F}$ & F21 & 10 & $\mathrm{~F}$ \\
\hline T22 & 10 & $\mathrm{~F}$ & F22 & 10 & $\mathrm{~F}$ \\
\hline T23 & 10 & $\mathrm{~F}$ & F23 & 10 & $\mathrm{~F}$ \\
\hline T24 & 10 & $\mathrm{~F}$ & F24 & 9 & $\mathrm{~F}$ \\
\hline B3 & 10 & $\mathrm{~F}$ & B3 & 10 & $\mathrm{~F}$ \\
\hline T31 & 10 & $\mathrm{~F}$ & F31 & 10 & $\mathrm{~F}$ \\
\hline T32 & 10 & $\mathrm{~F}$ & F32 & 10 & $\bar{F}$ \\
\hline T33 & 10 & $\mathrm{~F}$ & F33 & 10 & $\mathrm{~F}$ \\
\hline T34 & 10 & $\mathrm{~F}$ & F34 & 10 & $\mathrm{~F}$ \\
\hline
\end{tabular}

Table (24): Evaluation of prepared corrosion inhibitors morpholino(thiophen-2-yl)methanone II and morpholino(furan-2-yl)methanone IV with standard commercial alkyd resin

\begin{tabular}{|c|c|c|c|c|c|}
\hline \multirow{2}{*}{ Resin Code } & \multicolumn{2}{|l|}{ Blistering } & \multirow{2}{*}{ Resin Code } & \multicolumn{2}{|l|}{ Blistering } \\
\hline & Size & Frequency & & Size & Frequency \\
\hline ST & 10 & $\mathrm{~F}$ & ST & 10 & $\mathrm{~F}$ \\
\hline ST+ $0.5 \%$ II & 10 & $\mathrm{~F}$ & ST+ $0.5 \%$ IV & 10 & $\mathrm{~F}$ \\
\hline ST+ 1\% II & 10 & $\mathrm{~F}$ & $\mathrm{ST}+1 \% \mathrm{IV}$ & 10 & $\mathrm{~F}$ \\
\hline $\mathrm{ST}+3 \%$ II & 10 & $\mathrm{~F}$ & $\mathrm{ST}+2 \%$ IV & 10 & $\mathrm{~F}$ \\
\hline ST+ 5\% II & 10 & $\mathrm{~F}$ & $\mathrm{ST}+3 \% \mathrm{IV}$ & 10 & $\mathrm{~F}$ \\
\hline
\end{tabular}

Table (25): Evaluation of prepared corrosion inhibitors morpholino(thiophen-2-yl)methanone " II " and morpholino(furan-2-yl)methanone " IV" with some prepared modified alkyd resin (synergism)

\begin{tabular}{|c|c|c|c|c|c|}
\hline \multirow{2}{*}{ Resin Code } & \multicolumn{2}{|l|}{ Blistering } & \multirow{2}{*}{ Resin Code } & \multicolumn{2}{|l|}{ Blistering } \\
\hline & Size & Frequency & & Size & Frequency \\
\hline T32 & 10 & $\mathrm{~F}$ & F32 & 10 & $\mathrm{~F}$ \\
\hline T32+ $0.5 \%$ II & 10 & $\mathrm{~F}$ & F32+ $0.5 \%$ IV & 10 & $\mathrm{~F}$ \\
\hline T32+ 1\% II & 10 & $\mathrm{~F}$ & F32+ 1\% IV & 7 & $\mathrm{M}$ \\
\hline T32+3\% II & 10 & $\mathrm{~F}$ & F32+ 2\% IV & 10 & $\mathrm{~F}$ \\
\hline T32+ 5\% II & 10 & $\mathrm{~F}$ & F32+ 3\% IV & 5 & $\mathrm{MD}$ \\
\hline
\end{tabular}

10 : means no blistering (the best) 0 : the worst blistering degree

F: few M: medium MD: medium dense 
The following photos of tested films after 240 hours in $5 \%$ NaCL solution are shown below:

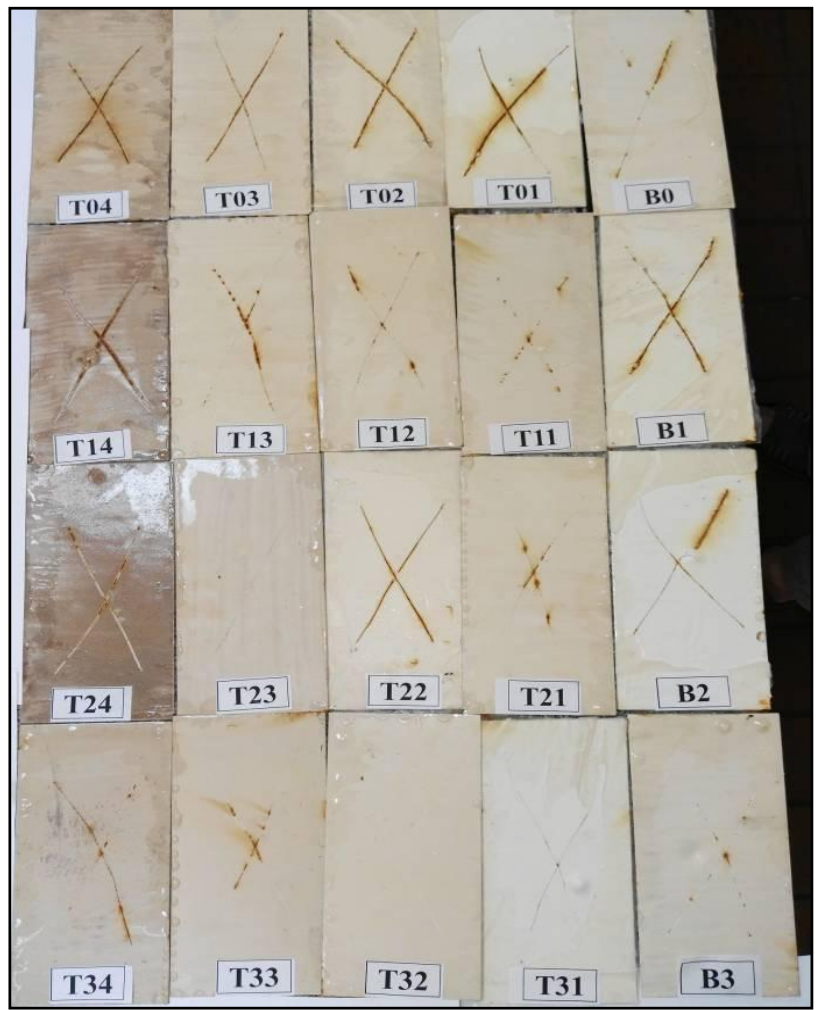
Fig. (9): ZnPO4 primer with HETCA-modified alkyd resins
resins

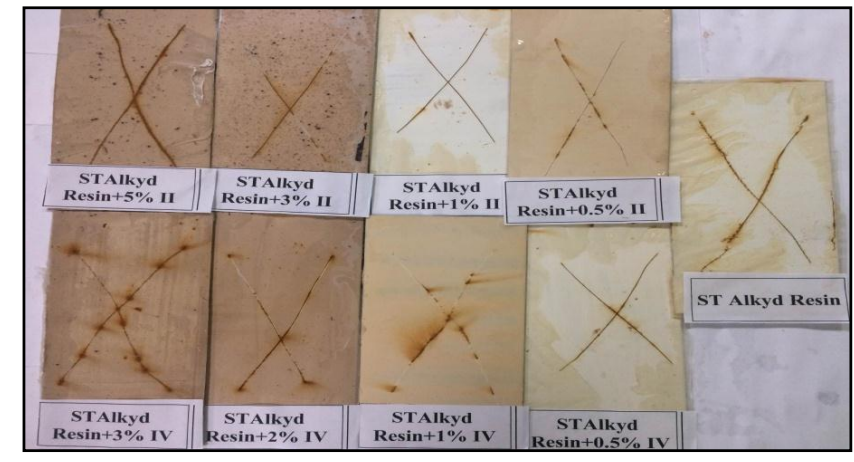

Fig. (11): ZnPO4 primer with standard commercial alkyd resins with prepared corrosion inhibitors II \& IV.

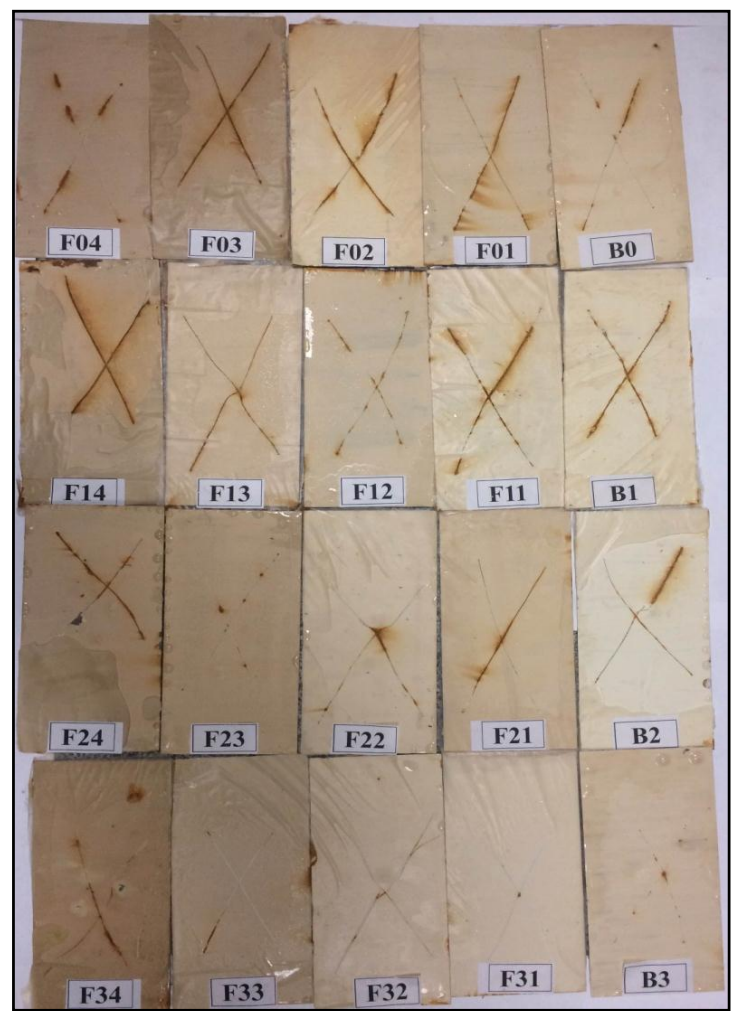

Fig. (10): ZnPO4 primer with HEFCA-modified alkyd

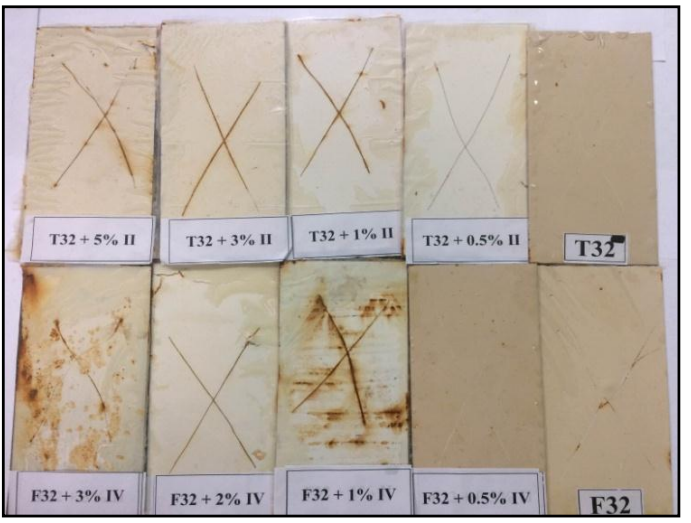

Fig. (12): ZnPO4 primer with $T 32$ and $F 32$ alkyd resins and prepared corrosion inhibitors II and IV.
0B : Removed area is greater than $65 \%$, $1 \mathrm{~B}: 35-65 \%$ removed area, $2 \mathrm{~B}: 15-35 \%$ removed area, $3 \mathrm{~B}: 5-15 \%$ removed area,

The corrosion resistance increases by the increase of excess-OH percent of both modified and unmodified alkyd resins.

There is a certain modification percent of alkyd resins at which such alkyd resin achieves maximum corrosion resistance, and this optimum modification decreases by increasing excess-OH \% of alkyd resin.

For example:
At $0 \%$ excess-OH alkyd resins (their Blank sample is B0) the maximum corrosion resistance achieves at $20-30 \%$ modification (i.e. T03 and F04)

At $10 \%$ excess-OH alkyd resins (their Blank sample is B01) the maximum corrosion resistance achieves at 5-20\% modification (i.e. T11 and F12)

At 20\% excess-OH alkyd resins (their Blank sample is B02) the maximum corrosion resistance achieves at 5-20\% modification (i.e. T23 and F23)

At $10 \%$ excess-OH alkyd resins (their Blank sample is B01) the maximum corrosion resistance 
achieves at $5-10 \%$ modification (i.e. T32 and F31)

In other words, for achieving the maximum corrosion resistance of alkyd resins, the need for modification will decrease by increasing excess-OH percent.

The addition of prepared compounds II and IV achieves corrosion resistance effect and the optimum dosage of the addition will be as follow:

1- Morpholino(thiophen-2-yl)methanone II is $1 \%$ on total formula weight

2- Morpholino(furan-2-yl)methanone IV is $0.5 \%$ on total formula weight

The addition of prepared corrosion inhibitors II and IV on some modified alkyd resins such as T32 and F32, respectively, improves their corrosion resistance and gives additional protection (SYNERGISM) for the metal substrate at a certain dosage $0.5 \%$ on total formula weight .

\section{Conclusions}

The following observations were noticed during the investigation:

1- All resins prepared are very clear and transparent and homogenous

2- The color of modified alkyd resins is very dark due to the entering of nitrogen atom in their chains.

3- The reaction rate for various resins increases by the increasing of the percentage of modifier and also increases with the increase of excess-OH percentage.

4- The viscosity for various resins at constant solid content increases slightly by the increasing of the percentage of modifier, but increases dramatically with the increase of excess-OH percentage.

5- The drying time increases by increasing the modifier percentage and by decreasing excess-OH percent of alkyd resins. There are some difficulties in the drying of lower excess$\mathrm{OH} \%$ alkyd resins, and also, in higher modi- fied alkyd resins due to the presence of Soya bean fatty acids in their structure.

6- All dried films are excellent water, acid, solvent resistance

7- Only the higher excess-OH percent alkyd resins are good alkali resistant.

8- The HETCA- and HEFCA-modification increases the alkali resistance of alkyd resins.

9- All films passed the adhesion and flexibility test except the higher modified alkyd resins in adhesion tests.

10-The HETCA and HEFCA modification increases the corrosion resistance performance of alkyd resins at certain optimum values.

11- The prepared Morpholino(thiophen-2-yl) methanone II and Morpholino(furan-2-yl)methanone IV are corrosion inhibitors additives and they can improve the corrosion resistance of HETCA- and HEFCA- modified alkyd resins.

\section{REFERENCES}

1. ISH Chemical Estimates, SRI Alkyd Surface Coatings (2013)

2. I.A. Sabbah, F. Abd El-Hai and M.Y. Gabr, Preparation and evaluation of some modified alkyd resins for surface coatings, Al-Azhar Bull. Sci, pp. 129-136, March (2010)

3. M.A. Abd El Ghaffar, E.A.M. Youssef, M.M.H. Abo Shosa, N.A. Ibrahim, Modified alkyd resin as an anticorrosive binder for paints, Pigment \& Resin Technology, Vol. 25 Iss: 6, pp.5-9 (1996)

4. K. Vaso, M. Dema, Preparation of the anticorrosive paints with long oil alkyd resins modified with maleic anhydride and phthalic anhydride, ZAŠTITA MATERIJALA 51 No. 2 (2010)

5. Ayman M. Atta, Rasha A. El-Ghazawy1 and Ashraf M. ElSaeed, Corrosion Protective Coating Based on Alkyd Resins Derived from Recycled Poly (ethylene terephthalate) Waste for Carbon steel, Int. J. Electrochem. Sci., Vol. 8, pp. $5136-5152$, (2013)

6. I.A. Sabbah, F. Abd El-Hai and M.Y. Gabr, Antimicrobial activity of some modified polyesteramide resins for surface coatings, Al-azhr Bull. Sci. J., Vol.22, No.2, pp. 1928. (2010).

7. H. Abd El-Wahabb, Various Modified Polyesteramide Varnishes as Antimicrobial Surface Coating Vehicles. Al-azhr Bull. Sci.J. vol.19, No.1, Jun., pp. 322-332, (2008).

8. T. C. Patton, Alkyd resin technology, formulating techniques and allied calculations, manual no. 8 , Ed. Interscience, New York, (1962) 